\title{
Strongly coupled quantum criticality with a Fermi surface in two dimensions: fractionalization of spin and charge collective modes
}

\author{
Subir Sachdevf and Takao Morinari闹 \\ Department of Physics, Yale University, P.O. Box 208120, New Haven CT 06520-8120
}

(Dated: July 4, 2002)

\begin{abstract}
We describe two dimensional models with a metallic Fermi surface which display quantum phase transitions controlled by strongly interacting critical field theories below their upper critical dimension. The primary examples involve transitions with a topological order parameter associated with dislocations in collinear spin density wave ("stripe") correlations: the suppression of dislocations leads to a fractionalization of spin and charge collective modes, and this transition has been proposed as a candidate for the cuprates near optimal doping. The coupling between the order parameter and long-wavelength volume and shape deformations of the Fermi surface is analyzed by the renormalization group, and a runaway flow to a non-perturbative regime is found in most cases. A phenomenological scaling analysis of simple observable properties of possible second order quantum critical points is presented, with results quite similar to those near quantum spin glass transitions and to phenomenological forms proposed by Schröder et al. (Nature 407, 351 (2000)).
\end{abstract}

\section{INTRODUCTION}

An important property of most quantum phase transitions studied to date in systems with a metallic Fermi surface in spatial dimensions $d \geq 2$ is that the critical field thepry for the order parameter is a free Gaussian theory 1.2. This result has its origin in the fact that the order parameters considered can be expressed as a fermion bilinears, and consequently the order parameter fluctuations are efficiently overdamped and suppressed by fermionic particle and hole excitations near the Fermi surface. The temperature dependencies of physical observables near the quantum sriticalpoint have been per-

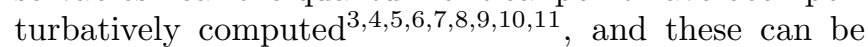
understood as corrections to scaling at the Gaussian critical point 12 .

The quantum and thermal fluctuations near quantum phase transitions in Fermi systems have been used to interpret experiments in a variety of correlated electron materials. In the cuprate superconductors, the anomalous properties in the normal state have been described in terms of the proximity to a quantum phase transition associated with the onset of spin density wave (SDW r $^{2}$ harge density wave (CDW) order in a Fermi liquid 13.425 . However, the observed anomalous properties extend to quite high temperatures, and it would be preferable to explain these in theories with stronger nonlinearities among the order parameter modes. Further, many of the anomalous properties extend to the optimal doping regime where there is no strong evidence of a large correlation length of the SDW and CDW orders.

This paper describes an alternative class of quantum critical points whose critical theories are non-Gaussian, and which remain strongly coupled even in the presence of a Fermi surface because they are below their upper critical dimension. Only in such theories can the order parameter relaxation rate, and possibly the quasiparticle energy width, be generically equal to $k_{B} T$ times a universal numerical constant 16 ( $T$ is the absolute temperature).
Examples of such quantum phase transitions abound in insulators and superconductors 128 . 19 , and some of these have been used to explain low-temperature properties of the cuprate superconductors20212223. This paper will discuss transition in metals with a Fermi surface, and so the possible regime of applicability is restricted to higher temperatures where superconductivity is absent.

Our primary focus will be on a transition with a nonlocal "topological" order associated with certain defects in the SDW/CDW order. However, long-range SDW or CDW order will not be present on either side of the critical point. This transition (shown in Fig. 目below) is being offered as a candidate23 24 for a possible optimal doping quantum critical point 25,26 in the cuprate superconductors. This proposal for the optimal doping quantum critical point must be distinguished from a conventional SDW ordering transition in a superconductor discussed recently by one of us22.23; the latter transition occurs at lower doping concentrations (near 1/8) and was used to predict and explain a number of recent low temperature neutron scattering and STM experiments. Here we are interested in higher dopings and temperatures, where, as we have already noted, the correlation length of conventional SDW/CDW order is surely quite small, but, as we shall discuss below, correlations in a topological order may be far more robust.

We also hope for applications of our theory in other correlated materials, like the heavy fermion systems, where many experiments are in disagreement with the conventional weakly coupled theories27.28. In particular, we will show that the strongly-coupled critical points predict a scaling structure for physical observables which is quite similar to those proposed by Schröder et al.29 Related scaling structures are also known to appear near strongly-coupled spin glass quantum critical points, and this is reviewed in Appendix A.

There is one simple route to obtaining a strongly coupled field theory in the presence of a Fermi surface that has been discussed earlierl: use restrictions from momentum conservation to prevent the order parameter from 
coupling linearly to low energy fermionic excitations. For example, a transition with the onset of SDW order at the wavevector $\mathbf{K}$, but the geometry of the Fermi surface such that no two points on the Fermi surface are separated by $\mathbf{K}$. In such a situation the order parameter excitations remain undamped, and the fermions appear to be innocuous bystanders to the transition. However, it turns out that the Fermi surface can still be relevant for the structure of the critical theory in certain situations; these effects are related to those that arise in the transitions we do discuss below, and so we defer discussion of this point until Section IV.

Turning to transitions characterized by a non-local, 'topological' order parameter, a situation that has been much discussed in the recent literature 30 has the electron, $c_{\varsigma}^{\dagger},(\varsigma=\uparrow, \downarrow$ is a spin index) fractionalize into a spinless charge $e$ boson, $b$, and a neutral spin $1 / 2$ fermion $f_{\varsigma}$ : $c_{\varsigma}^{\dagger}=b f_{\varsigma}^{\dagger}$ This fractionalization transition is described by a $Z_{2}$ gauge theory 31 , and both $b$ and $f_{\varsigma}$ carry a unit gauge charge; the physical fermion, $c_{\varsigma}^{\dagger}$, is, of course, gauge neutral. If we denote the $Z_{2}$ gauge field by $\sigma_{i j}(i, j$ are site labels), then terms like $\sigma_{i j} f_{i \uparrow} f_{j \downarrow}$ will generically appear in the effective Hamiltonian 30 . In the fractionalized phase, where the fluctuations of $\sigma_{i j}$ are effectively quenched, this term is a BCS-like pairing of the $f_{\varsigma}$ fermions, and it implies that the Fermi surface will generically be gapped out (except possibly at special points if the pairing is anisotropic). Consequently, this fractionalization transition does not provide us with a candidate quantum phase transition in the presence of a Fermi surface, and we will not consider it further in this paper.

The main focus of this paper shall be on a fractionalization transition that does less damage to the integrity of the electron: the electron (and hence its Fermi surface) remains intact on both sides of the transition, but charge neutral collective modes (in the particle-hole sector) do fractionalize; only at the quantum critical point, and in the associated non-zero temperature quantum critical region, is the electronic quasiparticle ill-defined. Zaanen et al.4 have recently given an appealing pictorial description of such a transition. A transition with closely related physical content was introduced in Ref. 23; we will follow the latter approach here, and will describe the physical content and experimental motivation below in Section I1. There have also been earlier discussions of the fractionaljzation of order parameters in other physical contexts 32.34.

After introducing the order parameters in Section II, we write down and classify quantum field theories for the critical points in Section III. We pause briefly in Section IV to consider the conventional transitions mentioned above, in which the fermion bilinear order parameter is not damped by Fermi surface excitations because of restrictions arising from momentum conservation, and show that it is described by field theories similar to those in Section III. The latter are subjected to a renormalization group analysis in Section $\mathrm{V}$, and the physical implications of the results are noted in Section VI.

\section{ORDER PARAMETER AND PHYSICAL MOTIVATION}

Consider a correlated electronic system in two dimensions with enhanced SDW correlations at the wavevectors $\mathbf{K}_{x}$ and $\mathbf{K}_{y}$; these are vectors of equal length pointing along the $x$ and $y$ axes respectively. Similar considerations apply to other choices for the ordering wavevectors, but we will focus on this case because it directly applies to the doped cuprates 35 at doping concentrations above 0.055. We can write for the spin operator $S_{\alpha}(\mathbf{r}, \tau)(\mathbf{r}$ is a spatial co-ordinate, $\alpha=x, y, z$ labels spin components, and $\tau$ is imaginary time):

$$
S_{\alpha}(\mathbf{r}, \tau)=\operatorname{Re}\left[e^{i \mathbf{K}_{x} \cdot \mathbf{r}} \Phi_{x \alpha}(\mathbf{r}, \tau)+e^{i \mathbf{K}_{y} \cdot \mathbf{r}} \Phi_{y \alpha}(\mathbf{r}, \tau)\right] ;
$$

$\Phi_{x \alpha}$ and $\Phi_{y \alpha}$ are then the SDW order parameters. Except of the case of two sublattice order $\left(\mathbf{K}_{x}=(\pi, 0)\right.$ or $(\pi, \pi))$, these order parameters are complex numbers. Concommitant with this SDW correlations, symmetry demands36, B that there are also "bond order" correlations at half the wavelength:

$$
\begin{aligned}
Q_{\mathbf{a}}(\mathbf{r}, \tau) & \equiv S_{\alpha}(\mathbf{r}, \tau) S_{\alpha}(\mathbf{r}+\mathbf{a}, \tau) \\
& \approx \operatorname{Re}\left[e^{2 i \mathbf{K}_{x} \cdot \mathbf{r}+i \mathbf{K}_{x} \cdot \mathbf{a}} \Phi_{x \alpha}^{2}(\mathbf{r}, \tau)\right]+\ldots
\end{aligned}
$$

there is an implied summation over the repeated spin in$\operatorname{dex} \alpha$. The vector a represents a bond (say the nearest neighbor vector), we have assumed that $\Phi_{x \alpha}$ does not vary significantly over the spatial distance $\mathbf{a}$, and the ellipses denote numerous other similar terms which can be deduced from (2.1). For a a nearest-neighbor vector, $Q_{\mathbf{a}}$ measures the modulations in the exchange energy, while for $\mathbf{a}=0, Q_{\mathbf{a}}$ measures the local charge density (for the $t$ - $J$ model the charge density is linearly related to the onsite $\left.S_{\alpha}^{2}\right)$. So, quite generally, the bond order parameters, $\Phi_{x, y \alpha}^{2}$, measure modulations in all observables invariant under spin rotations and time reversal (such the electron kinetic, pairing, or exchange energies). The modulation in the total charge density may be strongly suppressed by the long-range Coulomb interactions, but this suppression is not expected to apply to observables with $\mathbf{a} \neq 0$. We will loosely refer to such bond order modulations as CDW order in this paper, but the caveats discussed in this paragraph must be kept in mind.

Now examine the structure of the effective potential, $V\left(\Phi_{x \alpha}\right)$ controlling fluctuations of $\Phi_{x \alpha}$ (parallel considerations apply implicitly in the remainder of this subsection to $\left.\Phi_{y \alpha}\right)$. On general symmetry grounds23.36, this potential has the form

$$
\begin{aligned}
V\left(\Phi_{x \alpha}\right)=\widetilde{s} \Phi_{x \alpha}^{*} \Phi_{x \alpha}^{*} & +\frac{\widetilde{u}}{2} \Phi_{x \alpha}^{*} \Phi_{x \beta}^{*} \Phi_{x \alpha} \Phi_{x \beta} \\
& +\frac{\widetilde{v}}{2} \Phi_{x \alpha}^{*} \Phi_{x \alpha}^{*} \Phi_{x \beta} \Phi_{x \beta}+\ldots
\end{aligned}
$$

where $\widetilde{s}, \widetilde{u}, \widetilde{v}$ are phenomenological Landau parameters, and there is again an implied summation over repeated spin indices $\alpha, \beta$. In the usual Landau theory, the transition to the onset long-range SDW order happens when $\widetilde{s}$ 
first becomes negative, and the minimum of the potential moves from $\Phi_{x \alpha}=0$ to a non-zero value. The theory of the critical point then accounts for fluctuations of $\Phi_{x \alpha}$ about the value of $\Phi_{x \alpha}=0$, and these allow for strong fluctuations in both the amplitudes and the phases of the three complex fields $\Phi_{x \alpha}$.

In much of the discussion on stripe physics in the literature (see e.g. Ref. 24), the physical picture advocated for the onset of long range SDW/CDW (or "stripe") order is quite different. As one decreases the doping from a Fermi liquid towards a Mott insulator, initial local stripe-like correlations form, the the orientation and phase of these regions changes from point to point; only when these locally ordered regions align with each other, is there long range SDW/CDW order. In terms of our order parameter formulation, this means that locally the amplitude $\Phi_{x \alpha}^{*} \Phi_{x \alpha}$ first becomes large, and that long range order develops subsequently upon alignment of the orientation and phases of $\Phi_{x \alpha}$.

In the familiar $\varphi^{4}$ field theory of a real vector order parameter, this change in perspective from a "soft-spin" Landau theory (with strong amplitude fluctuations) to a "hard-spin" perspective (with amplitude fluctuations quenched) does not make a fundamental difference in the physics being considered. The orientation fluctuations in the hard-spin approach are described by a nonlinear $\sigma$ model, whose critical properties can be analyzed by expansion away the lower critical dimension. Conversely, the soft-spin approach leads to an expansion away from the upper critical dimension. However, these are merely complementary approaches to the same critical point, and the quantum numbers of the excitations in the phases flanking the critical point are the same in both approaches; indeed a $1 / N$ expansion ( $N$ is the number of field components) can interpolate between the soft-spin and hard-spin theories, and also between the upper and lower critical dimensions 38 .

For the present situation with a complex vector order parameter the situation is dramatically different, and there is a fundamental difference between the soft-spin and hard-spin approaches to fluctuations implied by the effective potential (2.3). The key difference is that not all order parameter configurations with a fixed $\Phi_{x \alpha}^{*} \Phi_{x \alpha}$ are physically equivalent, and they cannot all be rotated into each other by a symmetry transformation. A minimization of (2.3) at fixed $\Phi_{x \alpha}^{*} \Phi_{x \alpha}$ shows two distinct classes of minima, chosen by the sign of $\widetilde{v}$; their most general form is:

$$
\begin{array}{ll}
\text { (I) } \widetilde{v}>0 & : \Phi_{x \alpha}=n_{1 \alpha}+i n_{2 \alpha} \\
& \text { with } n_{1,2 \alpha} \text { real, } n_{1 \alpha}^{2}=n_{2 \alpha}^{2} \text { and } n_{1 \alpha} n_{2 \alpha}=0 \\
\text { (II) } \widetilde{v}<0 & : \Phi_{x \alpha}=e^{i \theta_{x}} n_{x \alpha} \\
& \text { with } n_{x \alpha} \text { real }
\end{array}
$$

By inserting into (2.1), it is easy to see that (I) describes a spiral SDW for which the charge order in (2.2) vanishes, while (II) is a collinear SDW with a modulation in the length of the spin as a function of $\mathbf{r}$, and a concommi- tant charge order. All experimental indications 39 are that the spin fluctuations in the cuprates are always of the form (II). It therefore seems physically reasonable to impose the amplitude constraint implied by (II) at an early stage, and to examine the theory associated with $V\left(\Phi_{x \alpha}\right)$ in the limit of large and negative $\widetilde{v}$ (stability requires $\widetilde{u}+\widetilde{v}>0)$. This last constraint is the more formal statement of the physical requirement that the spin and charge ordering is "stripe-like".

As has been discussed in Ref. 23, the imposition of the limit $\widetilde{v}<0$ at the outset leads to a theory for phase and orientation fluctuations with a non-trivial structure that is very naturally described by a $Z_{2}$ gauge theory. With the parameterization (II) in (2.4), and for fixed $\Phi_{x \alpha}^{*} \Phi_{x \alpha}$, the order parameter $\Phi_{x \alpha}$ takes values in the space $\left(S_{2} \times S_{1}\right) / Z_{2}$, where the $S_{2}$ pertains to the orientation of $n_{x \alpha}$, the $S_{1}$ is the phase factor $41 e^{i \theta_{x}}$, while the all-important $Z_{2}$ quotient accounts for the invariance of $\Phi_{x \alpha}$ under $n_{x \alpha} \rightarrow n_{x \alpha}$ and $\theta_{x} \rightarrow \theta_{x}+\pi$. We can define global actions 23 for single-valued fields $n_{x \alpha}$ and $e^{i \theta_{x}}$ only at the cost of introducing a $Z_{2}$ gauge field $\sigma_{i j}= \pm 1$. A simple phenomenological form for the spatial terms in such an action is33:

$$
\begin{aligned}
\mathcal{S}_{Z_{2}} & =-\frac{1}{g_{\theta}} \sum_{\langle i j\rangle} \sigma_{i j} \cos \left(\theta_{x i}-\theta_{x j}\right)-\frac{1}{g_{n}} \sum_{\langle i j\rangle} \sigma_{i j} n_{x \alpha i} n_{x \alpha j} \\
& +K \sum_{\square} \prod_{\square} \sigma_{i j},
\end{aligned}
$$

where $i, j$ are lattice sites, and $g_{\theta}, g_{n}$, and $K$ are coupling constants that vary with doping. The $Z_{2}$ gauge invariance ensures that all physical properties are invariant under the $Z_{2}$ gauge transformation $\theta_{x i} \rightarrow \theta_{x i}+\left(1-\sigma_{i}\right) \pi / 2$, $n_{x \alpha i} \rightarrow n_{x \alpha i} \sigma_{i}$ and $\sigma_{i j} \rightarrow \sigma_{i} \sigma_{i j} \sigma_{j}$. The point defects associated with order parameter space $\left(S_{2} \times S_{1}\right) / Z_{2}$ were analyzed in Ref. 23, and a fundamental role is played by the "stripe dislocation", sketched in Fig 1, which is a half-vortex in the angular field $\theta_{x}$. This vortex also carries a $Z_{2}$ gauge flux of -1 , as is easily seen from (2.5).

We are now in a position to introduce our candidate transition for strongly coupled quantum criticality in the presence of a Fermi surface. Begin in a Fermi liquid state, with only short range spin and charge order correlations: $\left\langle\Phi_{x \alpha}\right\rangle=0,\left\langle\Phi_{x \alpha}^{2}\right\rangle=0\left(\widetilde{s}\right.$ in (2.3) is large and positive, $g_{\theta}$, $g_{n}$ in (2.5) are not large) and only a small core energy for the stripe dislocations, which therefore proliferate. The dislocation core energy is controlled by the Maxwell term for the $Z_{2}$ gauge field, $K$, and a small value of $K$ implies that the $Z_{2}$ gauge field is in its confining state. In this state, the strong fluctuations of the $Z_{2}$ gauge field "bind" the $n_{x \alpha}$ and $e^{i \theta_{x}}$ fluctuations together, and the appropriate collective excitation is the conventional field $\Phi_{x \alpha}$ itself, as it appears in (2.1). The single $\Phi_{x \alpha}$ collective mode therefore describes both the SDW fluctautions (via 2.1) ) and the bond order/CDW fluctuations (via (2.2)).

Now increase the dislocation core energy by increasing $K$, while keeping $\widetilde{s}$ reasonably large (or $g_{\theta}, g_{n}$ small): this will decrease the number of stripe dislocations but still 


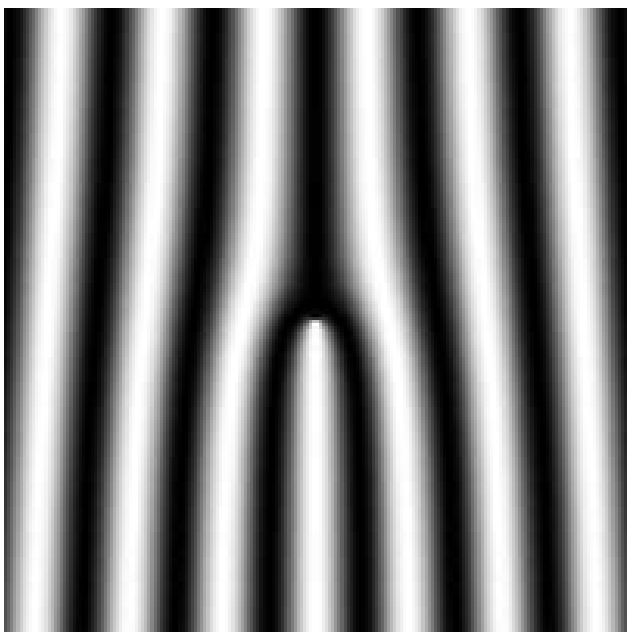

FIG. 1: A stripe dislocation: gray scale plot of $\delta \rho(\mathbf{r}) \sim$ $\cos \left(2 \mathbf{K}_{x} \cdot \mathbf{r}+2 \theta_{x}\right)$ (see (2.2) and (2.4)) with $\theta_{x}=\pi / 2+$ $(1 / 2) \tan ^{-1}(y / x)$ containing a half vortex. As noted below (2.2) $\delta \rho(\mathbf{r})$ should not be literally interpreted as the electron charge density, and the modulation about could be associated with e.g. the exchange per link. The quantum phase transitions discussed in this paper involve a transition from a Fermi liquid state in which these dislocations proliferate to one in which they are suppressed; there is no long-range spin or charge density wave order in either state (see also Fig. Q).

maintain $\left\langle\Phi_{x \alpha}\right\rangle=0,\left\langle\Phi_{x \alpha}^{2}\right\rangle=0$. In the cuprates we imagine that the increase in $K$ is associated with the decrease in carrier concentration towards the Mott insulator. At a critical value of $K_{c}$, the $Z_{2}$ gauge theory undergoes a transition into a deconfined state in which the stripe dislocations are suppressed. The transition at $K=K_{c}$ is the focus of interest in this paper. In this deconfined state, the $n_{x \alpha}$ and $e^{i \theta_{x}}$ fluctuations become independent collective excitations: i.e. the single spin/charge collective mode, $\Phi_{x \alpha}$, has fractionalized into two independent modes. The bond order, (2.2), fluctuations are now given by the 'square' of the $e_{x}^{i \theta}$ collective mode, while the spin fluctuations, (2.1), are the product of the $n_{x \alpha}$ and $e^{i \theta_{x}}$ collective modes.

We reiterate that there is no long range spin or charge density wave order on either side of this fractionalization transition, and it is entirely associated with the suppression of the defects in Fig 11; see Fig 2. At dopings lower than those shown in Fig 2, the cuprates possess phases with a variety of possible long range orders (e.g. SDW or CDW) which were reviewed in Ref. 23. These transitions are associated with conventional order parameters, and we will not consider them in this paper, as theories are already available in the literature.

\section{QUANTUM FIELD THEORIES}

We are interested in describing the universal properties of the transition at $K=K_{c}$. These turn out to be

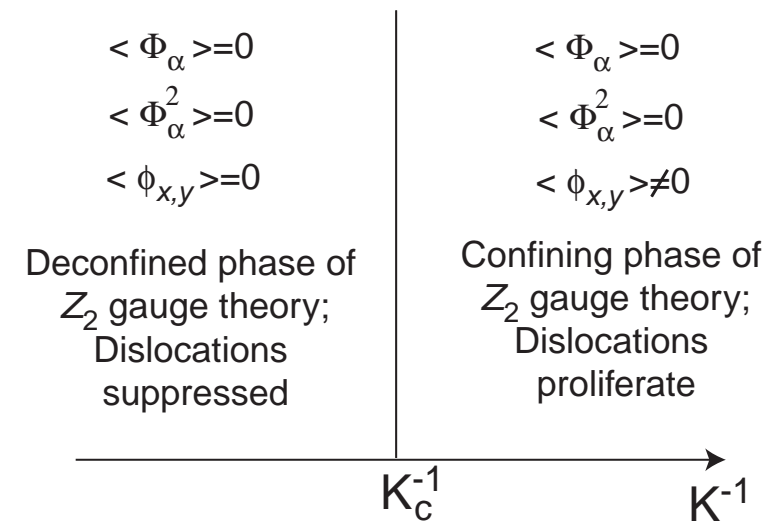

FIG. 2: The quantum phase transition at $K=K_{c}$ is the one studied in this paper. The phases on both sides of $K_{c}$ have well defined electronic quasiparticles near a Fermi surface and no long range spin or charge order $\left(\left\langle\Phi_{x, y \alpha}\right\rangle=0,\left\langle\Phi_{x, y q}^{2}\right\rangle=0\right)$. The order parameters $\phi_{x, y}$ are defined in Section III these are Ising order parameters dual to the $Z_{2}$ gauge theory. The phase on the right is a conventional Fermi liquid; that on the left is a Fermi liquid with local stripe correlations with random phases and spin orientations, and a 'topological rigidity' associated with the suppression of stripe dislocations. In the application to the cuprates, the horizontal axis represents increasing doping and the critical point is near optimal doping. States with long range spin or charge density wave order do appear at smaller doping but these have not been shown. The phase transition at $K=K_{c}$ should be distinguished from the conventional SDW ordering transition in a superconductor; thiofours at lower doping and was used recently by one of us2 23 to predict and explain the results of neutron scattering and STM experiments.

controlled by two distinct characteristics of the electronic system.

(i) Tetragonal or orthorhombic crystal symmetry: With tetragonal symmetry we have to simultaneously consider the separate $Z_{2}$ gauge theories associated with fractionalization transitions in both $\Phi_{x \alpha}$ and $\Phi_{y \alpha}$. With orthorhombic symmetry, only one of these will be selected (the "stripes" have a preferred direction).

(ii) Presence or absence of long-range Coulomb interactions: As we argue below, an important coupling of the critical $Z_{2}$ gauge degrees of freedom to the fermions arises from long-wavelength deformations of the shape of the Fermi surface. As is well known, in the presence of longrange Coulomb interactions the "dilatational" mode, involving local changes in the area of the Fermi surface, is strongly suppressed. For completeness we will also consider the case of fermions with only short-range interactions (perhaps the Coulomb interactions have been screened by a metallic gate), where the coupling to the dilatational mode has to be included.

With these two criteria, four distinct categories emerge, which we label A, B, C, D, as summarized in Table I. We will describe these cases in the subsections below. 
TABLE I: Classification of quantum field theories.

\begin{tabular}{c|c|c|c}
\hline \hline Case & Conditions & Fields & Action \\
\hline A & $\begin{array}{c}\text { Orthorhomic symmetry and } \\
\text { long-range Coulomb interactions }\end{array}$ & $\phi_{x}$ & $\mathcal{S}_{x}$ \\
\hline B & $\begin{array}{c}\text { Tetragonal symmetry and } \\
\text { long-range Coulomb interactions }\end{array}$ & $\phi_{x}, \phi_{y}, \psi_{I}$ & $\mathcal{S}_{x y}+\mathcal{S}_{I}$ \\
\hline C & Orthorhombic symmetry and & $\phi_{x}, \psi$ & $\mathcal{S}_{x}+\mathcal{S}_{\psi}$ \\
& short-range interactions & & \\
\hline D & Tetragonal symmetry and & $\phi_{x}, \phi_{y}$, & $\mathcal{S}_{x y}+\mathcal{S}_{I}$ \\
& short-range interactions & $\psi_{I}, \psi$ & $+\mathcal{S}_{\psi}^{\prime}$ \\
\hline \hline
\end{tabular}

\section{A. Case A}

We begin with case A, for which the critical theory has the simplest form. As we noted in Section II, the transition at $K=K_{c}$ is described by a $Z_{2}$ gauge theory. We know that the pure $2+1$ dimensional $/$ gauge theory is dual a $2+1$ dimensional Ising model 30.42 43; we represent this Ising order parameter by a real scalar field $\phi_{x}$. For now, we neglect the independent $Z_{2}$ gauge theory associated with $\Phi_{y \alpha}$, because orthorhombic symmetry has oriented all the spin and charge density waves along $\Phi_{x \alpha}$. The action for $\phi_{x}$ in the vicinity of the transition is the familiar field theory for the Ising critical point:

$\mathcal{S}_{x}=\int d^{2} r d \tau\left[\frac{1}{2}\left(\partial_{\tau} \phi_{x}\right)^{2}+\frac{1}{2}\left(\nabla_{r} \phi_{x}\right)^{2}+\frac{s}{2} \phi_{x}^{2}+\frac{u}{24} \phi_{x}^{4}\right] ;$

we have chosen the spatial units so that the velocity of $\phi_{x}$ excitations is unity in both directions, $u$ is a quartic non-linearity, and the quadratic coupling $s \sim K-K_{c}$ is the tuning parameter across the transition. The field $\phi_{x}$ can be viewed as the creation/annihilation operator for the dislocations: $\phi_{x}$ is condensed in the confining phase where the dislocations proliferate, and $\phi_{x}$ has short-range correlations in the deconfined phase where dislocations are suppressed.

In principle, we also need to account for the "matter" fields $\theta_{x}, n_{x \alpha}$ in (2.5) near this confinement transition. Closely related $Z_{2}$ or plge models have been considered in the literature 30.3243 , and it has been established that their fluctuations do not change the universality class of the transition. Their presence does modify the effective couplings in (3.1), but the confinement transition continues to be described by the Ising field theory (3.1) all along the phase boundary.

The main question we need to address here is the coupling between $\phi$ and the electronic excitations near the Fermi surface; the gapless excitations near the Fermi surface have the potential of inducing long-range interactions which are more destructive than the matter fields in (2.5). The electrons do not carry any Ising gauge charges, and couple to degrees of freedom of interest here only via the bilinears in (2.1) and (2.2):

$$
-\lambda_{1} \sum_{\mathbf{r}} c_{\varsigma}^{\dagger}(\mathbf{r}) \tau_{\varsigma \varsigma^{\prime}}^{\alpha} c_{\varsigma^{\prime}}(\mathbf{r}) S_{\alpha}(\mathbf{r})-\lambda_{2} \sum_{\mathbf{r}} c_{\varsigma}^{\dagger}(\mathbf{r}) c_{\varsigma}(\mathbf{r}) Q_{\mathbf{a}}(\mathbf{r}),
$$

where $\tau^{\alpha}$ are the spin Pauli matrices. We now imagine integrating out $\Phi_{x \alpha}$ and generating terms which couple the electrons to the $Z_{2}$ gauge degrees of freedom. The electrons are gauge singlets, and so can only couple to the physical $Z_{2}$ gauge flux: the simplest such coupling is one in which the coupling $K$ in (2.5) becomes $\mathbf{r}, \tau$ dependent via its dependence on the local fermion bilinears

$$
(K, s) \rightarrow(K, s)+\lambda_{3} c_{\varsigma}^{\dagger}(\mathbf{r}) c_{\varsigma}(\mathbf{r})+\lambda_{4} c_{\varsigma}^{\dagger}(\mathbf{r}) \nabla_{\mathbf{r}}^{2} c_{\varsigma}(\mathbf{r})+\ldots,
$$

where the ellipses represent terms with higher derivatives; we have to include all such terms because the typical electron momentum is of order the Fermi momentum, and this is not small. Upon performing the duality transition to the Ising field $\phi_{x}$, the mapping applies to $s$, the co-efficient of $\phi_{x}^{2}$, via its dependence upon $K$, and this is also noted in (3.3). We can now integrate over the fermionic degrees of freedom and examine the structure of the terms which modify $\mathcal{S}_{x}$ : it is not difficult to see that apart from the modification of the numerical values of the couplings in (3.1), all such terms are formally irrelevant. The leading non-analytic terms induced by the low energy fermionic excitations arise from long wavelength deformations of the Fermi surface: in the presence of long-range Coulomb interactions, all such excitations allowed by symmetry to couple to $\phi_{x}^{2}$ are suppressed. So we reach the important conclusion that the critical theory for case A is specified by (3.1).

There is one important caveat to this conclusion that deserves highlighting. Our analysis has neglected topological Berry phase terms that could be present in the coupling between $\phi_{x}$, the fermions, and the $Z_{2}$ gauge field $\sigma_{i j}$. Similar phase factors do arise in other analyses of $Z_{2}$ gauge theories 30 . We leave the study of this issue to future work, and restrict our attention here to couplings associated with (3.3).

\section{B. Case B}

The higher tetragonal symmetry has two important consequences: we have two consider fractionalization in both $\Phi_{x \alpha}$ and $\Phi_{y \alpha}$ simultaneously, and distortions of the Fermi surface with $B_{1}$ symmetry do couple to the critical degrees of the freedom even in the presence of long-range Coulomb interactions.

There are now two dual Ising fields, $\phi_{x}$ and $\phi_{y}$, associated with the independent $Z_{2}$ gauge fields in the $x$ and $y$ directions; simple symmetry considerations show that 
$\mathcal{S}_{x}$ in (3.1) is replaced by

$$
\begin{aligned}
\mathcal{S}_{x y}=\int d^{2} r d \tau & {\left[\frac{1}{2}\left(\partial_{\tau} \phi_{x}\right)^{2}+\frac{1}{2}\left(\partial_{\tau} \phi_{y}\right)^{2}+\frac{1}{2}\left(\nabla_{r} \phi_{x}\right)^{2}\right.} \\
+ & \frac{1}{2}\left(\nabla_{r} \phi_{y}\right)^{2}+\frac{s}{2}\left(\phi_{x}^{2}+\phi_{y}^{2}\right) \\
+ & \left.\frac{u}{24}\left(\phi_{x}^{2}+\phi_{y}^{2}\right)^{2}+\frac{v}{4} \phi_{x}^{2} \phi_{y}^{2}\right]
\end{aligned}
$$

for simplicity, we have neglected a possible difference in the velocity of the $\phi_{x}$ modes in the $x$ and $y$ directions. The critical properties of the theory $\mathcal{S}_{x y}$ are well understood 44 : they are described by a stable fixed point with $v^{*}=0$ and a global $\mathrm{O}(2)$ (or XY) symmetry of rotations between $\phi_{x, y}$.

The fundamental new phenomenon here is that low energy deformations of the Fermi surface destabilize the critical point just noted. Although long-range Coulomb interactions do suppress a local uniform dilatational change in the Fermi surface, they do not suppress shape deformations which preserve the local volume. In particular, the tetragonal symmetry allows an oscillation of the Fermi surface with $B_{1}\left(\right.$ or $\left.d_{x^{2}-y^{2}}\right)$ symmetry which has singular contributions at low energy as has been emphasized recently by Oganesyan et al 10 ; moreover, by symmetry, this deformation will couple linearly to $\phi_{x}^{2}-\phi_{y}^{2}$. Integrating the Fermi surface deformations out by a standard procedure 10 , we obtain the following non-analytic term in the effective action:

$$
\lambda_{5} \int \frac{d^{2} q}{(2 \pi)^{2}} \int \frac{d \omega}{2 \pi} \frac{|\omega|}{|\mathbf{q}|}\left|\phi_{x}^{2}(\mathbf{q}, \omega)-\phi_{y}^{2}(\mathbf{q}, \omega)\right|^{2} ;
$$

the $|\omega| /|\mathbf{q}|$ factor is the standard density of states of long-wavelength Fermi surface oscillations in dimensions $\geq 2$. A simple scaling argument now gives the scaling dimension of the coupling $\lambda_{5}$ at the $v^{*}=0$ fixed point of $\mathcal{S}_{x y}$. The $|\omega| /|\mathbf{q}|$ factor implies that (3.5) couples $\left(\phi_{x}^{2}(\mathbf{r}, \tau)-\phi_{y}^{2}(\mathbf{r}, \tau)\right)$ to itself at distinct spacetime points, which precludes any additional operator renormalization; consequently

$$
\operatorname{dim}\left[\lambda_{5}\right]=d+1-2 \operatorname{dim}\left[\phi_{x}^{2}-\phi_{y}^{2}\right]
$$

here and henceforth we will state such scaling dimensions for general spatial dimensions $d$, although our interest is in $d=2$. The scaling dimension of $\phi_{x}^{2}-\phi_{y}^{2}$ can in turn be related to the anisotropy crossover exponent 4.4 , $\phi_{T}$

$$
\operatorname{dim}\left[\phi_{x}^{2}-\phi_{y}^{2}\right]=d+1-\frac{\phi_{T}}{\nu}
$$

where $\nu$ is the correlation length exponent of the $v^{*}-0$ fixed point of $\mathcal{S}_{x y}$. Using the known numerical values 45,46 of these exponents in $d=2, \phi_{T}=1.184$ and $\nu=0.67155$, we obtain $\operatorname{dim}\left[\lambda_{5}\right]=0.52>0$. So we have established our earlier claim that $B_{1}$ deformations of the Fermi surface constitute a relevant perturbation.
It is now necessary to extend the renormalization group to include contributions higher order in $\lambda_{5}$. However, a simple one-loop analysis shows that this is not a stable procedure 47: at order $\lambda_{5}^{2}$ we generate additional quartic terms which have a different $\omega, \mathbf{q}$ dependence than that in (3.5), and new relevant terms are generated at each successive order. The solution to this conundrum is to introduce a new real scalar field, $\psi_{I}$, as a HubbardStratonovich decoupling of (3.5): this field will be a measure of the amplitude of $B_{1}$ deformations of the Fermi surface. Note that we expect $\lambda_{5}>0$, and so to make the Hubbard-Stratonovich transformation positive definite, it is necessary to subtract a $\left(\phi_{x}^{2}-\phi_{y}^{2}\right)^{2}$ term before decoupling, and to compensate for this by corresponding changes to $u, v$. In this manner we get the action

$$
\begin{gathered}
\mathcal{S}_{I}=\frac{1}{2} \int \frac{d^{2} q}{(2 \pi)^{2}} \int \frac{d \omega}{2 \pi}\left(1+a_{I} \frac{|\omega|}{|\mathbf{q}|}\right)\left|\psi_{I}(\mathbf{q}, \omega)\right|^{2} \\
-\frac{g}{2} \int d^{2} r d \tau \psi_{I}\left(\phi_{x}^{2}-\phi_{y}^{2}\right) ;
\end{gathered}
$$

our final effective action for case B is $\mathcal{S}_{x y}+\mathcal{S}_{I}$. In obtaining $\mathcal{S}_{I}$ we have expanded the coefficient of $\psi_{I}^{2}$ for small $\lambda_{5} \sim a_{5}$, and rescaled $\psi_{I}$ to make the co-efficient of the analytic $\psi_{I}^{2}$ term unity. We now have two new coupling constants, $g$ and $a_{I}$, and it can be checked that $\lambda_{5}>0$ implies $a_{I}>0$. Further, at the fixed points of $\mathcal{S}_{x y}$, we will show in Section $\mathrm{VA}$ that $\operatorname{dim}\left[a_{I}\right]=\operatorname{dim}\left[\lambda_{5}\right]$.

The most important property of $\mathcal{S}_{x y}+\mathcal{S}_{I}$ is that this action is stable under renormalization, and no new relevant terms are generated at any order. Consequently, it can be subjected to a standard field-theoretic renormalization group analysis, and this will be presented in Section $\square$.

\section{Case C}

The analysis of the remaining cases is a straightforward generalization of the discussion in Section IIIB.

As in Case A, the orthorhombic symmetry implies that we need only one Ising field $\phi_{x}$. However, because of the absence of long-range Coulomb interactions, dilatational changes in the local Fermi surface volume with full lattice symmetry do carry large low energy spectral weight. We measure these changes by the real scalar field $\psi$, and obtain its effective action

$$
\begin{gathered}
\mathcal{S}_{\psi}=\frac{1}{2} \int \frac{d^{2} q}{(2 \pi)^{2}} \int \frac{d \omega}{2 \pi}\left(1+a \frac{|\omega|}{|\mathbf{q}|}\right)|\psi(\mathbf{q}, \omega)|^{2} \\
-\frac{g}{2} \int d^{2} r d \tau \psi \phi_{x}^{2} .
\end{gathered}
$$

The effective action for case $\mathrm{C}$ is now $\mathcal{S}_{x}+\mathcal{S}_{\psi}$. An argument similar to that in Section III B can be used to compute the scaling dimension of $a$ : as in (3.6,3.7) we find that the fixed point describing the transition of $\mathcal{S}_{x}$

$$
\operatorname{dim}[a]=\frac{2}{\nu}-d-1=\frac{\alpha}{\nu}
$$


where $\alpha, \nu$ are the standard exponents of the $2+1$ dimensional Ising model. Using their known valueste, we obtain $\operatorname{dim}[a]=0.174>0$. So the fixed point of case A is unstable to a Fermi surface with short-range interactions, and there is new universality class of critical behavior.

We note that the discussion in this subsection, with zero sound modes of a Fermi surface coupling to the square of an order parameter, bears a passing resemblance to a discussion of the influence of phonon modes in the superfluid state of bosons presented by Frey and Balent, 49

\section{Case D}

With tetragonal symmetry and no long-range Coulomb interactions, both dilatational and $B_{1}$ modifications of the Fermi surface have to be included. So the full effective action is $\mathcal{S}_{x y}+\mathcal{S}_{I}+\mathcal{S}_{\psi}^{\prime}$ where

$$
\begin{gathered}
\mathcal{S}_{\psi}^{\prime}=\frac{1}{2} \int \frac{d^{2} q}{(2 \pi)^{2}} \int \frac{d \omega}{2 \pi}\left(1+a \frac{|\omega|}{|\mathbf{q}|}\right)|\psi(\mathbf{q}, \omega)|^{2} \\
-\frac{g}{2} \int d^{2} r d \tau \psi\left(\phi_{x}^{2}+\phi_{y}^{2}\right)
\end{gathered}
$$

At the $v^{*}=0$ fixed point of $\mathcal{S}_{x y}$, we can show, as in Section III , that $\operatorname{dim}[a]=\alpha / \nu$, where $\alpha, \nu$ are now critical exponents of the $2+1$ dimensional XY model. Numerically 46 we have $\operatorname{dim}[a]=-0.022<0$, and so $a$ is irrelevant at this fixed point. However, the perturbations in $\mathcal{S}_{I}$ remain relevant, and we cannot a priori conclude that the terms in $\mathcal{S}_{\psi}^{\prime}$ will also be irrelevant at any possible stable fixed point of $\mathcal{S}_{x y}+\mathcal{S}_{I}$; so it is necessary to include (3.11) in the analysis of Case D.

\section{CONVENTIONAL ORDER PARAMETERS WITHOUT FERMI SURFACE DAMPING}

This section makes a brief detour to a separate class of quantum phase transitions mentioned in Section il: those with conventional order parameters which do not couple linearly to low energy excitations on the Fermi surface. We will consider here a simple example of such a transition, although the results discussed here are far more general: an SDW ordering transition with the order parameters defined as in (2.1), from a Fermi liquid state with $\left\langle\Phi_{x, y \alpha}\right\rangle=0$ to an SDW state with $\left\langle\Phi_{x, y \alpha}\right\rangle \neq 0$. Further, we assume that the values of $\mathbf{K}_{x, y}$ are such that they cannot connect any two points on the Fermi surface; consequently the $\Phi_{x, y \alpha}$ fluctuations are undamped and the quantum critical modes are strongly coupled. The effective action for $\Phi_{x, y \alpha}$ will have a structure similar to that of $\mathcal{S}_{x}, \mathcal{S}_{x y}$ in (3.1), (3.4), apart from changes in the quartic terms which are similar to those in (2.3).

It should now be clear that the zero momentum composite fields $\left|\Phi_{x, y \alpha}\right|^{2}$ can couple to long-wavelength deformations of the Fermi surface in much the same way as the composite fields $\phi_{x, y}^{2}$ did in Section [II. For orthorhombic symmetry and short-range interactions (as in Case C), only $\Phi_{x \alpha}$ is critical (say), and its coupling to the long-wavelength particle-hole continuum leads to the term

$$
\lambda_{6} \int \frac{d^{2} q}{(2 \pi)^{2}} \int \frac{d \omega}{2 \pi} \frac{|\omega|}{|\mathbf{q}|}\left|\Phi_{x \alpha}^{2}(\mathbf{q}, \omega)\right|^{2},
$$

which is the analog of (3.5). Just as in (3.9.3.10)

$$
\operatorname{dim}\left[\lambda_{6}\right]=\frac{\alpha}{\nu},
$$

and so the coupling in (4.1) is relevant if and only if $\alpha>0$. Most conventional order parameters have $\alpha<0$ in $2+1$ dimensions, in which case the Fermi surface has no influence on the critical theory. The main exception is the Ising case, found e.g. at a two sublattice CDW ordering transition, in which case the theory will identical to that in Section III C.

For a system with tetragonal symmetry, there is stronger coupling to quadrupolar distortions of the Fermi surface, just as discussed in Section IIIB. The analog of (3.5) is the term

$$
\left.\lambda_{7} \int \frac{d^{2} q}{(2 \pi)^{2}} \int \frac{d \omega}{2 \pi} \frac{|\omega|}{|\mathbf{q}|}|| \Phi_{x \alpha}\right|^{2}(\mathbf{q}, \omega)-\left.\left|\Phi_{y \alpha}\right|^{2}(\mathbf{q}, \omega)\right|^{2},
$$

and the scaling dimension of $\lambda_{7}$ is related to an anisotropy crossover exponent; this always such that $\lambda_{7}$ is strongly relevant. The analysis of the resulting theory will be similar to Case B above, although we will not present it explicitly.

\section{RENORMALIZATION GROUP ANALYSIS}

This section returns to the collective mode fractionalization transitions considered in Sections III and III; closely related analyses will apply to the cases mentioned in Section IV.

Case A is described by the purely bosonic theory (3.1), whose properties are very well known: its critical point is in the universality class of the $2+1$ dimensional Ising model. So we move directly to case B in the first subsection, and mention the straightforward generalization to the other cases later.

\section{A. Case B}

Renormalization group (RG) analyses of models with a structure similar to $\mathcal{S}_{x y}+\mathcal{S}_{I}$ have appeared elsewhere in the literature (see e.g. Ref. 49), and so we can omit most of the technical details. We consider the rescaling transformation

$$
r=r^{\prime} e^{\ell} \quad ; \quad \tau=\tau^{\prime} e^{z \ell}
$$


under which the fields $\phi_{x, y}, \psi_{I}$ transform as

$$
\begin{aligned}
\phi_{x, y}(r, \tau) & =e^{-(d+z-2+\eta) \ell / 2} \phi_{x, y}^{\prime}\left(r^{\prime}, \tau^{\prime}\right) \\
\psi_{I}(r, \tau) & =e^{-(d+z+\theta) \ell / 2} \psi_{I}^{\prime}\left(r^{\prime}, \tau^{\prime}\right)
\end{aligned}
$$

here $z, \theta$, and $\eta$ are $\ell$-dependent parameters to be determined by loop corrections to the effective action under the $\mathrm{RG}$ transformation - the values of these parameters at any fixed point of the RG equations will specify the corresponding critical exponents. The one-loop graphs have the same topology as those considered in Ref. 49, and we defer details on the evaluation of the loop integrals to Appendix B; the results are

$$
\begin{aligned}
\frac{d a_{I}}{d \ell} & =(1-z-\theta) a_{I} \\
\frac{d g}{d \ell} & =\frac{(4-d-z-\theta-2 \eta)}{2} g-\left(\frac{u}{3}-\frac{v}{2}\right) g \mathcal{I}\left(0, a_{I}\right) \\
& +g^{3} \mathcal{I}\left(1, a_{I}\right) \\
\frac{d u}{d \ell} & =(4-d-z-2 \eta) u-\frac{\left(10 u^{2}+6 u v+9 v^{2}\right)}{6} \mathcal{I}\left(0, a_{I}\right) \\
& +6 u g^{2} \mathcal{I}\left(1, a_{I}\right)-6 g^{4} \mathcal{I}\left(2, a_{I}\right) \\
\frac{d v}{d \ell} & =(4-d-z-2 \eta) v-\frac{\left(4 u v+3 v^{2}\right)}{2} \mathcal{I}\left(0, a_{I}\right) \\
& -\frac{2(4 u+3 v)}{3} g^{2} \mathcal{I}\left(1, a_{I}\right) \\
\theta & =-g^{2} \mathcal{I}\left(0, a_{I}\right) \\
\eta & g^{2} \mathcal{J}\left(a_{I}\right) \\
z & =1+\frac{g^{2} \mathcal{K}\left(a_{I}\right)-\eta}{2}
\end{aligned}
$$

where $\left.\mathcal{I}_{(} m, a_{I}\right)$ ( $m$ integer), $\mathcal{J}\left(a_{I}\right)$, and $\mathcal{K}\left(a_{I}\right)$ are scheme dependent functions which are specified in Appendix B. We have assumed that the strongly relevant "mass" term $s$ has been tuned to its critical value, and are examining above only the flow within this critical manifold. The first of the equations in (5.3) is the most important: it is an exact equation (although the values of $z$ and $\theta$ do have corrections at higher order) which follows from the fact that there are no loop corrections to the non-analytic term with coefficient $a_{I}$ in the effective action.

First, let us examine (5.3) in the subspace with $a_{I}=0$, where the long-range forces associated with the Fermi surface are absent. The results in Appendix 5.3) show that $\mathcal{I}(m, 0)=\mathcal{C}$ independent of $m$, and $\mathcal{J}(0)=\mathcal{K}(0)=$ 0 ; here $\mathcal{C}$ is a scheme-dependent constant whose value does not effect the physical properties. It is then easy to search for fixed points of (5.3): there are 8 fixed points, and all of them obey $z=1$ (this is exact to all orders for
$\left.a_{I}=0\right)$ and $\eta=0$ (to this order). The fixed points are:

$$
\begin{array}{ll}
\left(G_{1}\right) \quad u^{*}=0 ; v^{*}=0 ; g^{* 2}=0 \\
\left(G_{2}\right) \quad u^{*}=\frac{3 \epsilon}{\mathcal{C}} ; v^{*}=-\frac{2 \epsilon}{\mathcal{C}} ; g^{* 2}=\epsilon \\
\left(C_{1}\right) \quad u^{*}=\frac{\epsilon}{3 \mathcal{C}} ; v^{*}=\frac{2 \epsilon}{9 \mathcal{C}} ; g^{* 2}=0 \\
\left(C_{2}\right) \quad u^{*}=\frac{10 \epsilon}{3 \mathcal{C}} ; v^{*}=-\frac{16 \epsilon}{9 \mathcal{C}} ; g^{* 2}=\epsilon \\
\left(X Y_{1}\right) \quad u^{*}=\frac{3 \epsilon}{5 \mathcal{C}} ; v^{*}=0 ; g^{* 2}=0 \\
\left(X Y_{2}\right) \quad u^{*}=\frac{12 \epsilon}{5 \mathcal{C}} ; v^{*}=-\frac{6 \epsilon}{5 \mathcal{C}} ; g^{* 2}=\frac{3 \epsilon}{5 \mathcal{C}} \\
\left(I_{1}\right) \quad u^{*}=\frac{2 \epsilon}{3 \mathcal{C}} ; v^{*}=-\frac{2 \epsilon}{9 \mathcal{C}} ; g^{* 2}=0 \\
\left(I_{2}\right) \quad u^{*}=\frac{5 \epsilon}{3 \mathcal{C}} ; v^{*}=-\frac{8 \epsilon}{9 \mathcal{C}} ; g^{* 2}=\frac{\epsilon}{3 \mathcal{C}}
\end{array}
$$

where $\epsilon=3-d$. The physical meaning of these fixed points becomes clear after integrating out the $\psi_{I}$ field in $\mathcal{S}_{x y}$ at $a_{I}=0$ : for this value of $a_{I}$ this yields an effective action with the same structure as $\mathcal{S}_{x y}$ in (3.4) but with modified coupling constants

$$
\begin{aligned}
& \bar{u}=u-3 g^{2} \\
& \bar{v}=v+2 g^{2} .
\end{aligned}
$$

The reader can now observe that the fixed points in (5.4) all separate into pairs with equal values of $\bar{u}$ and $\bar{v}$, and our notation for the fixed points has been chosen to make this evident: $G_{1}$ and $G_{2}$ form one such pair, and similarly for the remainder.

At this som we can compare results with the analysis of Aharony 445 of the consequences of a cubic anisotropy on fixed points with $\mathrm{O}(n)$ symmetry: he analyzed the analog of the model $\mathcal{S}_{x y}$ for $n$-component fields, but with a somewhat different notation. The fixed points (5.4) are in complete agreement with his results: $G_{1,2}$ are the Gaussian fixed points, $C_{1,2}$ are the "cubic" fixed points, $X Y_{1,2}$ are the $\mathrm{XY}$ fixed points with $\mathrm{O}(2)$ symmetry and no cubic anisotropy, and $I_{1,2}$ are the Ising fixed points at which the fields $\phi_{1,2}$ are decoupled. As shown by Aharony, an XY fixed point is stable for the case of a two-component field being considered here.

Here, we have to perform some further analysis to distinguish between the two possible $X Y$ fixed points: these two have the same values of $\bar{u}, \bar{v}$, but only one has $g^{*} \neq 0$. A standard computation shows that fixed point $X Y_{2}$ with $g^{*} \neq 0$ is the only stable fixed point among all those in (5.4). The theory prefers the fixed point with $g^{*} \neq 0$ because the fluctuations of $\psi_{I} \sim \phi_{1}^{2}-\phi_{2}^{2}$ are characterized by a divergent susceptibility at the critical point 51 .

We are now in a position to consider non-zero values of $a_{I}$. For small $a_{I}$, the above analysis shows that near the fixed point $X Y_{2}$

$$
\frac{d a_{I}}{d \ell}=\frac{3 \epsilon}{5} a_{I}
$$




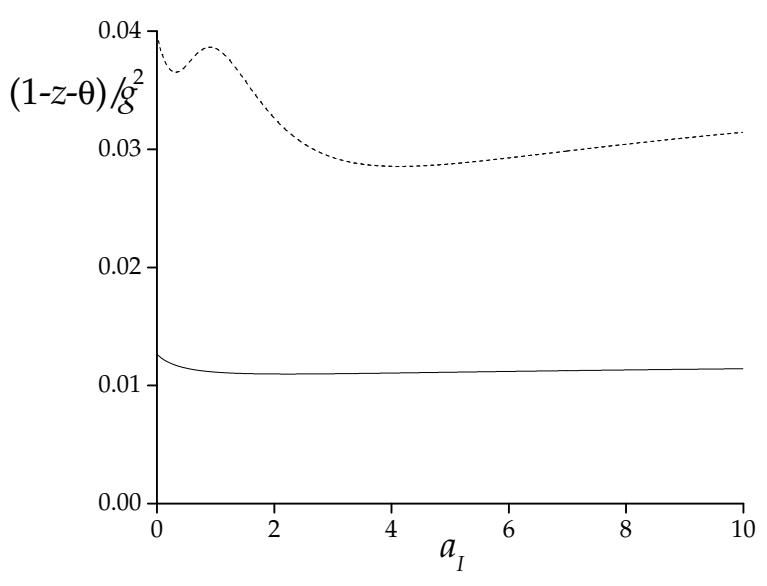

FIG. 3: Plot of $(1-z-\theta) / g^{2}$ as determined by (5.3). There is no zero crossing, and hence no finite, positive, non-zero $a_{I}$ fixed point. The full line is the minimal subtraction scheme, while the dashed line is the fixed dimension scheme.

In other words, $a_{I}$ is a relevant perturbation with $\operatorname{dim}\left[a_{I}\right]=3 \epsilon / 5$. We argued in Section IIIB that $\operatorname{dim}\left[a_{I}\right]=2 \phi_{T} / \nu-d-1$, and it can be checked that this is consistent with our present result and the $\epsilon$ expansion result 44 for $\phi_{T} / \nu$.

For general $a_{I}$, we have to consider the complete flow equations in (5.3). While these are quite complicated, it is clear from the first flow equation that a finite and nonzero $a_{I}$ fixed point, $a_{I}=a_{I}^{*}$ can only exist if and only if there is a solution to $1-z-\theta=0$. From (5.3) we observe that at the one loop order, this condition translates into an equation for $a_{I}^{*}$ alone:

$$
2 \mathcal{I}\left(0, a_{I}^{*}\right)+\mathcal{J}\left(a_{I}^{*}\right)-\mathcal{K}\left(a_{I}^{*}\right)=0 .
$$

Using the results in Appendix B, we plot half the left hand side of this equation in Fig 3 for two different renormalization schemes. No solution is found in either case, or in the additional cutoff schemes discussed in Appendix $B$. The value of $(1-z-\theta)$ does initially decrease from its value at $a_{I}=0$ which led to (5.6), but this decrease is not strong enough to lead to a zero crossing. So we have not found a finite $a_{I}$ fixed point, but we cannot rule out the possibility that one will appear upon including terms higher order in $g, u, v$.

It is clear now that any non-zero, positive $a_{I}$ runs away to $a_{I}=\infty$, and we have to consider the theory in this limit. An examination of the limiting values of the results in Appendix B showed that there were no finite fixed point values for $u, v$ in the limit of large $a_{I}$ either. In principle, it was possible that both $a_{I}$ and $g$ became large in a manner that permitted $u$ and $v$ to approach a finite fixed point value. However simple analytic and numerical analyses of (5.3) showed that this was not the case, and we always had flows to strong coupling for $u$ and $v$ also. Starting close to the stable fixed point at $a=0$, we found that the flows sent $v \rightarrow \infty$ and $u \rightarrow-\infty$. This is possibly suggestive of a first-order transition, but

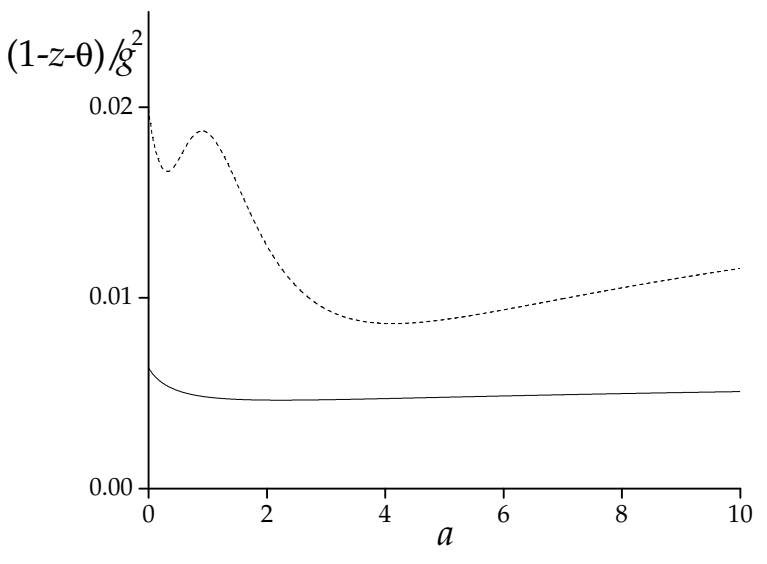

FIG. 4: An in Fig 3, but for (5.9).

no definitive conclusion can be reached because the RG equations themselves breakdown once $u$ or $v$ become of order unity.

\section{B. Case $\mathrm{C}$}

The analysis and results for Case $\mathrm{C}$ are similar to those for Case B above, and so we will be brief.

The RG flow equations (5.3) are replaced by

$$
\begin{aligned}
\frac{d a}{d \ell} & =(1-z-\theta) a \\
\frac{d g}{d \ell} & =\frac{(4-d-z-\theta-2 \eta)}{2} g-\frac{u g}{2} \mathcal{I}(0, a) \\
& +g^{3} \mathcal{I}(1, a) \\
\frac{d u}{d \ell} & =(4-d-z-2 \eta) u-\frac{3 u^{2}}{2} \mathcal{I}(0, a) \\
& +6 u g^{2} \mathcal{I}(1, a)-6 g^{4} \mathcal{I}(2, a) \\
\theta & =-\frac{g^{2}}{2} \mathcal{I}(0, a) \\
\eta & =g^{2} \mathcal{J}(a) \\
z & =1+\frac{g^{2} \mathcal{K}(a)-\eta}{2},
\end{aligned}
$$

where the functions $\mathcal{I}, \mathcal{J}, \mathcal{K}$ remain the ones specified in Appendix B. These equations do have fixed points at $a=0$ which have been specified completely in earlier work 51 . However, the stable fixed point for $a=0$ is unstable to a non-zero $a$, and the condition for a finite and non-zero $a$ fixed point is (replacing (5.7)):

$$
\mathcal{I}\left(0, a^{*}\right)+\mathcal{J}\left(a^{*}\right)-\mathcal{K}\left(a^{*}\right)=0 .
$$

The left hand side of this equation is plotted in Fig. 4, and again there is no solution. We find instead flows to strong coupling, as in Case B.

Finally, we note that the flow equations and results for Case $\mathrm{D}$ are a combination of those presented already for Cases $\mathrm{C}$ and $\mathrm{D}$, and we will not present them explicitly. 


\section{PHYSICAL PROPERTIES}

This section considers some simple observable physical properties of the fractionalization transition described in Section III. We will find that the properties of many observables are quite similar to another case with an order parameter which is a composite of the electron spin operator: the onset of spin glass order. To help make this comparison, we review the physical properties of the quantum spin glass transition in Appendix A.

Our analysis of collective mode fractionalization theories has found only one stable fixed point: that for case $A$ with orthorhombic symmetry and long-range Coulomb interactions. The other cases exhibited flows to strong coupling. This section will study the general scaling properties of various physical observables for case A, and also extrapolate the results to possible strongly coupling fixed points for the other cases.

\section{A. Dynamic spin susceptibility}

We focus on the spin susceptibility near the wavevectors $\mathbf{K}_{x, y}$; other susceptibilities of conventional order parameters can be treated in a similar way. The order parameters associated with these susceptibilities, $\Phi_{x, y \alpha}$, are controlled by an effective action of the form $\left(\omega_{n}\right.$ is a Matsubara frequency at a temperature $T$ )

$$
\mathcal{S}_{\Phi}=\frac{T}{2} \sum_{\omega_{n}} \int \frac{d^{2} q}{(2 \pi)^{2}}\left(\Gamma\left|\omega_{n}\right|+A \mathbf{q}^{2}+B\right)\left|\Phi_{x \alpha}\left(\mathbf{q}, \omega_{n}\right)\right|^{2}
$$

along with a similar term with $(x \rightarrow y)$. Unlike the situation in Section IV, we are considering the case where $\mathbf{K}_{x, y}$ do connect two points on the Fermi surface, and hence there is over-damping of $\Phi_{x, y \alpha}$ fluctuations represented by the term proportional to $\Gamma$. Also, the $\Phi_{x, y \alpha}$ fluctuations remain non-critical with $\left\langle\Phi_{x, y \alpha}\right\rangle=0$ at the fractionalization transition, and hence the parameter $B>0$.

We now have to consider the coupling of $\Phi_{x, y \alpha}$ to the critical modes $\phi_{x, y}$. As noted at the end of Section III A, we ignore possible non-local topological terms, and focus on the simplest local couplings allowed by symmetry; for the cases with tetragonal symmetry (cases B and D), these are

$$
\begin{aligned}
& \int d^{2} r d \tau {\left[\zeta_{1}\left(\left|\Phi_{x \alpha}\right|^{2}+\left|\Phi_{y \alpha}\right|^{2}\right)\left(\phi_{x}^{2}+\phi_{y}^{2}\right)\right.} \\
&+\zeta_{2}\left(\left|\Phi_{x \alpha}\right|^{2}-\left|\Phi_{y \alpha}\right|^{2}\right)\left(\phi_{x}^{2}-\phi_{y}^{2}\right)
\end{aligned}
$$

while for orthorhombic symmetry we merely drop the non-critical $\phi_{y}$ field above. We will consider the influence of the critical $\phi_{x, y}$ modes on $\Phi_{x, y \alpha}$ in a perturbation theory in $\zeta_{1}$. For case A, where a stable fixed point was found, such a procedure appears reasonably safe. The validity for the other cases, with flows to strong coupling, has a greater uncertainty and this should be kept in mind below.
The influence of the critical fluctuations on the noncritical $\Phi_{x, y \alpha}$ susceptibility will be determined by two (and higher) point correlators of the operators $\phi_{x}^{2}-\phi_{y}^{2}$ and $\phi_{x}^{2}+\phi_{y}^{2}$ for tetragonal symmetry, and $\phi_{x}^{2}$ alone for orthorhombic symmetry. Let us represent these operators generically by $\mathcal{O}$ and its scaling dimension by

$$
\operatorname{dim}[\mathcal{O}]=d_{\mathcal{O}}
$$

For case A, we have only $\mathcal{O}=\phi_{x}^{2}$ and $d_{\mathcal{O}}=d+1-1 / \nu$, where $\nu$ is the exponent of $2+1$ dimensional Ising model; for other cases, the value of $d_{\mathcal{O}}$ will be determined by the hypothetical strong-coupling fixed point. In perturbation theory in $\zeta_{1,2}$ we can write for the dynamic spin susceptibility

$$
\chi_{\Phi}(\mathbf{q}, \omega)=\frac{1}{-i \Gamma \omega+A \mathbf{q}^{2}+B+M(\mathbf{q}, \omega)}
$$

with the "self energy"

$$
M\left(\mathbf{q}, \omega_{n}\right)=\zeta^{2} T \sum_{\epsilon_{n}} \int \frac{d^{2} p}{(2 \pi)^{2}} D_{\mathcal{O}}\left(\mathbf{p}, \epsilon_{n}\right) \chi_{\Phi}\left(\mathbf{p}+\mathbf{q}, \epsilon_{n}+\omega_{n}\right)
$$

where $\zeta$ is related to $\zeta_{1,2}$, and $D_{\mathcal{O}}$ is the two-point correlator of $\mathcal{O}$. We are interested in the behavior of $M$ at small $\mathbf{q}$ and $\omega$; after inserting (6.4) into (6.5), we use the fact that $B>0$ to expand the momentum and frequency dependence of $\chi_{\Phi}$ in powers of $1 / B$. For the $\mathbf{q}$ dependence of $M$, a simple power-counting analysis shows that all terms in this series are free of infrared divergences as long as $d_{\mathcal{O}}>0$, in which case the $\mathbf{q}$ dependence of $M$ is analytic and free of critical singularities. Case A easily satisfies $d_{\mathcal{O}}>0$, and we expect this is also true for the other cases, because a negative $d_{\mathcal{O}}$ would imply that correlations of $\mathcal{O}$ increase with increasing spacetime distance.

In contrast to its $\mathbf{q}$ dependence, the $\omega$ dependence of $M$ is singular even for $d_{\mathcal{O}}>0$. This is most easily computed by analytically continuing (6.5) to real frequencies and taking its imaginary part. A standard analysis then shows that $M(\mathbf{q}, \omega)$ has a $\mathbf{q}$ independent singular contribution in the quantum critical region which obeys the scaling form

$$
M(\mathbf{q}, \omega) \sim T^{\left(2 d_{\mathcal{O}}+z\right) / z} F_{1}(\omega / T)
$$

(we are using $\hbar=k_{B}=1$ ), where $F_{1}$ is a universal scaling function determined by the non-trivial interacting fixed point of the strongly-coupled quantum field theories considered in Section III]; we expect $\operatorname{Im} F_{1}(y \rightarrow 0) \sim y$ and $F_{1}(y \rightarrow \infty) \sim \operatorname{sgn}(y)|y|^{\left(2 d_{\mathcal{O}}+1\right) / z}$. Specifically, $F_{1}$ is related to the local correlator of $\mathcal{O}$, which has a singular contribution obeying the scaling form

$$
\int \frac{d^{2} p}{(2 \pi)^{2}} D_{\mathcal{O}}\left(\mathbf{p}, \omega_{n}\right) \sim T^{2 d_{\mathcal{O}} / z} F_{2}\left(\omega_{n} / T\right),
$$

(the scaling function $F_{2}$ is a property of the stronglyinteracting fixed point of the quantum field theories of 
Section [II]) by the integral relation

$$
\begin{aligned}
& \operatorname{Im}\left[F_{1}(y)\right] \\
= & \int_{0}^{y} \frac{d \Omega}{\pi} \Omega \operatorname{Im}\left[F_{2}(y-\Omega)\right](1+n(\Omega)+n(y-\Omega)) \\
+ & \int_{0}^{\infty} \frac{d \Omega}{\pi} \Omega \operatorname{Im}\left[F_{2}(\Omega+y)\right](n(\Omega)-n(\Omega+y)) \\
+ & \int_{y}^{\infty} \frac{d \Omega}{\pi} \Omega \operatorname{Im}\left[F_{2}(\Omega-y)\right](n(\Omega-y)-n(\Omega)),(6
\end{aligned}
$$

where $n(\Omega)=1 /\left(e^{\Omega}-1\right)$ is the Bose function at unit temperature. Note that the leading factor of $\Omega$ in (6.8) arises from the linearly density of states of low frequency excitations implied by (6.4), upon ignoring the influence of $M$ in $\chi_{\Phi}$ on the right hand side of (6.5). This is permissible as long as the contribution of $M$ here is subdominant: from (6.6) we see that this is so as long as $d_{\mathcal{O}}>0$. If this inequality is violated, then the perturbative expansion in $\zeta$ fails, and a self consistent approach appears necessary.

It is interesting to note that the results $(6.4,6.6)$ imply a structure for the dynamic spin susceptibility which is closely related to phenomenological forms that have been proposed recently by Schröder et al. 2 to describe neutron scattering measurements on $\mathrm{CeCu}_{6-x} \mathrm{Au}_{x}$. Elegant theoretical models of a "local quantum criticality" based on the self-consistent treatment of quantum impurity ('Konde')medels have also been advanced52 53.54 and discussed 27 55 56.57 as explanations for the observations of Schröder et al.. Someimportant differences between the theories of $\mathrm{Si}$ et al 53.54 and our work here should be noted: underlying our results is a bona fide $d+1$ dimensional quantum field theory (presented in Section III), and not a quantum impurity model. Our theory leads to a $\mathbf{q}$-independent singular contribution to the dynamic spin susceptibility because $B>0$, as shown above. The condition $B>0$ also implies the static spin susceptibility is not divergent, in contrast to impurity model analyses in which it does diverge at the impurity critical point 53 . An associated fact is that the exponent in (6.6) is likely to satisfy $\left(2 d_{\mathcal{O}}+z\right) / z>1$, although this need not be true for the spin glass transitions discussed in Appendix A. It has also been noted55 57.58 that the divergence of the local susceptibility could preempt a local treatment of the impurity model critical point 53.54 .

It should be clear that the above mechanism for 'local' quantum criticality in the dynamic spin susceptibility is quite a general property of a situation in which the primary order parameter, which exhibits strongly coupled bulk criticality, is coupled to the spin operator only with non-linear terms like those in (6.2). As an illustration we recall in Appendix A that related considerations apply to the quantum spin glass transition.

\section{B. Fermion self energy}

We follow the same perturbative approach discussed in Section VIA. Singular fluctuations of $\mathcal{O}$ will couple to the electrons via couplings like

$$
\zeta_{3} \int d^{2} r d \tau \mathcal{O}(\mathbf{r}, \tau) c_{\varsigma}^{\dagger}(\mathbf{r}, \tau) c_{\varsigma}(\mathbf{r}, \tau)+\ldots ;
$$

the ellipses represent additional terms with gradients of the electron operators (these are not small because the typical electron momentum resides on the Fermi surface).

We should note at the outset that the status of a perturbative computation of the electron self energy, $\Sigma$, remains open. For the case of overdamped fermion critical points below their upper critical dimension, the forresponding issue addressed by Altshuler et al.59 and Castellani et al.60 who concluded that the leading perturbative correction also gave the ultimate critical singularity. The extension of this result to the strongly coupled critical points being discussed here is less clear. Indeed, for the case of a strongly coupled spin glass quantum critical point, the perturbative computation of the electron self energy is known to break down 61 (see also Appendix A).

With this caution in mind, we proceed with a computation of the consequences of (6.9). The electron self energy, $\Sigma$ to order $\zeta_{3}^{2}$ is

$$
\begin{aligned}
& \Sigma\left(\mathbf{q}, \omega_{n}\right)=\zeta_{3}^{2} T \sum_{\epsilon_{n}} \int \frac{d^{2} p}{(2 \pi)^{2}} D_{\mathcal{O}}\left(\mathbf{p}, \epsilon_{n}\right) \\
& \quad \times \frac{1}{i\left(\epsilon_{n}+\omega_{n}\right)-\varepsilon(\mathbf{p}+\mathbf{q})-\Sigma\left(\mathbf{p}+\mathbf{q}, \epsilon_{n}+\omega_{n}\right)},
\end{aligned}
$$

where $\varepsilon(\mathbf{p})$ is the electron dispersion. This expression can be evaluated using the methods discussed in e.g. Ref. 59. The result depends upon the scaling function associated with $D_{\mathcal{O}}$ for which we write

$$
D_{\mathcal{O}}(\mathbf{q}, \omega) \sim T^{\left(2 d_{\mathcal{O}}-d-z\right) / z} F_{3}\left(\omega / T, \mathbf{q} / T^{1 / z}\right),
$$

with $F_{3}$ a non-trivial universal scaling function determined by the strongly interacting fixed points of Section [II]. As in Ref. 59, the singular part of the self energy is momentum independent at this order, as long as $z>1$. Its frequency and temperature dependence is specified by the scaling form

$$
\Sigma(\mathbf{q}, \omega) \sim T^{\left(2 d_{\mathcal{O}}-1\right) / z} F_{4}(\omega / T),
$$

with the scaling function $F_{4}$ given by

$$
\begin{aligned}
\operatorname{Im}\left[F_{4}(y)\right] & =\int_{0}^{\infty} \frac{d \Omega}{\pi} \int_{0}^{\infty} k^{d-2} d k \operatorname{Im}\left[F_{3}(\Omega, k)\right] \\
& \times(2 n(\Omega)+f(\Omega-y)+f(\Omega+y)),
\end{aligned}
$$

where $n(\Omega)$ was defined below (6.8) and $f(\Omega)=1 /\left(e^{\Omega}+\right.$ $1)$ is the Fermi function at unit temperature.

\section{Transport}

We identify two distinct contributions to the critical electrical transport properties: the electronic quasiparti- 
cle and the bosonic collective modes. However, the cautions about a perturbative treatment of the coupling between these modes mentioned in Section VIB apply also to our discussion here.

The electronic contribution is characterized by the transport relaxation time $\tau_{\mathrm{tr}}$. The electron scattering discussed in Section VIB all occurs at small momenta $\mathbf{p}$ and, as is well known, the transport rate then acquires an additional factor of $\mathbf{p}^{2}$ over the quasiparticle scattering rate in the integral over scattered momenta. Extending the scaling arguments of Section VIB we see easily that

$$
\frac{1}{\tau_{\mathrm{tr}}} \sim T^{\left(2 d_{\mathcal{O}}+1\right) / z}
$$

The collective mode contribution is the analog of the "Aslamazov-Larkin" term in the theory of fluctuating superconductivity: fluctuations of the order parameters $\phi_{x, y}$ may themselves contribute to the electrical current J. The relationship between the dual fields $\phi_{x, y}$ and the microscopic degrees of freedom is extremely complex, and so we will restrict ourselves here to deducing the expressions for the current operator using symmetry arguments alone. The current $\mathbf{J}$ is odd under time reversal and spatial inversion, while the fields $\phi_{x, y}$ are even under both. Furthermore, the current should be invariant under the dual Ising symmetries involving the change in sign of $\phi_{x}$ and/or $\phi_{y}$. This indicates that the current operator has contributions from operators like

$$
J_{x} \sim \partial_{\tau} \phi_{x} \partial_{x} \phi_{x}, \phi_{x} \partial_{\tau} \partial_{x} \phi_{x}, \partial_{\tau} \phi_{y} \partial_{x} \phi_{y} \ldots
$$

and similarly for $J_{y}$. The scaling dimensions of these operators can be determined by standard methods for case A (some combination is related to components of the stress-energy tensor with scaling dimension $d+1$, but the others require a $(3-d)$ expansion), while for the other cases the scaling dimensions remain unknown quantities to be determined by the hypothetical strongcoupling fixed point. If we generically denote the scaling dimension of these operators by $d_{J}$, the Kubo formula implies that they will yield a singular contribution to the conductivity, $\sigma$, which scales as

$$
\delta \sigma \sim T^{\left(2 d_{J}-d-2 z\right) / z}
$$

Note that because the current is carried by both the electronic and bosonic degrees of freedom, we expect that we do not have to worry about constraints imposed by overall current conservation, as is the case in theories $\rightarrow f$ the superfluid-insulator transition in bosonic systems 16 .

\section{CONCLUSIONS}

This paper has described strongly interacting quantum phase transitions in the presence of a Fermi surface. The primary examples considered involve the phase transition described in Fig 2, from a conventional Fermi liquid state in which the dislocation in Fig 11 proliferates, to a 'topologically ordered' Fermi liquid state in which these dislocations have been suppressed. Note that there is no long-range SDW/CDW order in either state, although the amplitude of these orders must be locally large to allow identification of the dislocations. The conventional SDW/CDW orders are suppressed in both phases by 'spin-wave' fluctuations and by the proliferation of other defects, such as the hedgehogs in $n_{x \alpha}$ and integer vortices in $\theta_{x}$. We discussed some simple observable properties of such a topological phase transition in a Fermi liquid, and found results which were quite similar to those near quantum spin glass transitions, and to the phenomenolggical 'local' scaling forms proposed by Schröder et al.29. Despite their 'local' nature, the critical singularities are controlled by a bulk quantum field theory, and not a quantum impurity model.

The dislocation proliferation transition discussed here has been proposed as a candidate for a quantum critical point near optimal doping in the cuprate superconductors2324. In this context, it is interesting to note that recent scanning tunnelling microscopy images 62 of the surface of $\mathrm{Bi}_{2} \mathrm{Sr}_{2} \mathrm{CaCu}_{2} \mathrm{O}_{8+\delta}$ clearly show dislocations with the geometry of Fig 1 (see Fig 2c of Ref. 62).

The main shortcoming of our analysis here has been runaway flow of the renormalization group flow to a nonperturbative regime for the interesting cases. It is possible that this signals a more fundamental change in the nature of excitations near the Fermi surface than is accounted for by our formalism. We integrate out the low energy fermions at an early stage, and the strong coupling situation may benefit from an analysis in which quasiparticle degrees of freedom are retained more explicitly. Future work in this direction, along the lines of Refs. 63, appears desirable.

\section{Acknowledgments}

We thank A. Aharony, A. Chubukov, E. Demler, M. P. A. Fisher, M. Grilli, W. Metzner, A. Millis, V. Oganesyan, T. Senthil, Q. Si, M. Vojta, and J. Zaanen for useful discussions. This research was supported by US NSF Grant DMR 0098226. TM was supported by a Grant-in-Aid from the Ministry of Education, Culture, Sports, Science and Technology of Japan.

\section{APPENDIX A: QUANTUM SPIN GLASS TRANSITION}

The quantum spin glass transition in metallic systems bas been stydied in a number of recent works 61 64 65, 66 67, 68, 696. 70 . Here we review some physical properties whose scaling structure resembles the results in Section VI.

The central actor in the theory of the spin glass tran- 
sition is the order parameter functional

$$
Q_{\alpha \beta}^{a b}\left(\mathbf{r}, \tau_{1}, \tau_{2}\right) \sim S_{\alpha}^{a}\left(\mathbf{r}, \tau_{1}\right) S_{\beta}^{b}\left(\mathbf{r}, \tau_{2}\right)
$$

where $a, b$ are replica indices. In the quantum critical region, this functional has an expectation value of the form

$$
\left\langle Q_{\alpha \beta}^{a b}\left(\mathbf{r}, \tau_{1}, \tau_{2}\right)\right\rangle=\delta_{\alpha \beta} \delta^{a b} D\left(\tau_{1}-\tau_{2}\right)
$$

If the quantum critical point obeys hyperscaling properties, then Fourier transform of $D(\tau)$ will obey " $\omega / T$ scaling":

$$
D(\omega)=T^{\mu} F_{5}(\omega / T)
$$

It should be noted that the mean-field solution of the metallic spin glass does not obey (A3), and instead has a characteristic frequency scale $\sim T^{3 / 2}$. However, other mean-field spin glass models which accoun for proximity to a Mott insulator do obey $\omega / T$ scaling 61.64.67.68; These latter solutions have the value $\mu=0$, and $D(\omega)$ has the form

$$
\begin{gathered}
D(\omega) \propto \ln \left(\frac{\Lambda}{|\omega|}\right)-\int_{0}^{\infty} d \Omega \mathcal{P}\left(\frac{2 \Omega f(\Omega)}{\Omega^{2}-(\omega / T)^{2}}\right) \\
+\frac{i \pi}{2} \tanh \left(\frac{\omega}{2 T}\right)
\end{gathered}
$$

where $f(\Omega)$ was defined below (6.13) - for this value of $\mu$ there is an additive real contribution dependent upon the cutoff $\Lambda$, but the remainder is a universal function of $\omega / T$, and the function $D(\omega)$ is analytic as $\omega \rightarrow 0$ at any nonzero $T$. We will assume below that the lowdimensional spin-glass quantum critical point does obey the hyperscaling property A3, even if the mean-field theory for its particular situation does not.

We now consider the computations of the physical quantities discussed in Section VI. For the dynamic spin susceptibility near the wavevector $\mathbf{K}$, note that symmetry allows a coupling like

$$
\zeta_{4} \int d^{2} r d \tau_{1} d \tau_{2} Q_{\alpha \beta}^{a b}\left(\mathbf{r}, \tau_{1}, \tau_{2}\right) \operatorname{Re}\left[\Phi_{x \alpha}^{a *}\left(\mathbf{r}, \tau_{1}\right) \Phi_{x \beta}^{b}\left(\mathbf{r}, \tau_{2}\right)\right],
$$

along with the corresponding $(x \rightarrow y)$. Using the expectation value (A2) in A5, we immediately obtain a dynamic spin susceptibility of the form (6.4) with

$$
M(\mathbf{q}, \omega) \sim D(\omega)
$$

The singular part of the inverse susceptibility is $\mathbf{q}$ independent and a function of $\omega / T$, as in the local quantum criticality scenario. Again, however, the scaling function in (A3) is determined by a bulk $d$-dimensional quantum field theory.

Finally, we turn to the electron self energy, and the associated single particle and transport lifetimes. One approach, which follows Ref. 66, is to compute this perturbatively from the coupling which follows directly from the identification (A1

$$
\begin{gathered}
\zeta_{5} \int d^{2} r d \tau_{1} d \tau_{2} Q_{\alpha \beta}^{a b}\left(\mathbf{r}, \tau_{1}, \tau_{2}\right) c_{\varsigma}^{a \dagger}\left(\mathbf{r}, \tau_{1}\right) \tau_{\varsigma \varsigma^{\prime}}^{\alpha} c_{\varsigma^{\prime}}^{a}\left(\mathbf{r}, \tau_{1}\right) \\
\times c_{v}^{b \dagger}\left(\mathbf{r}, \tau_{2}\right) \tau_{v v^{\prime}}^{\alpha} c_{v^{\prime}}^{b}\left(\mathbf{r}, \tau_{2}\right) .
\end{gathered}
$$

Using A2, we can easily compute the electron self energy, $\Sigma$ perturbatively in $\zeta_{5}$, and at order $\zeta_{5}^{2}$ we find the contribution

$$
\Sigma\left(\mathbf{q}, \omega_{n}\right) \sim \zeta_{5}^{2} T \sum_{\epsilon_{n}} \operatorname{sgn}\left(\epsilon_{n}\right) D\left(\omega_{n}+\epsilon_{n}\right)
$$

which is momentum independent, but a singular function of frequency and temperature. Using (A3), we deduce that $\Sigma$ also obeys the scaling form

$$
\Sigma\left(\mathbf{q}, \omega_{n}\right) \sim T^{\mu+1} F_{6}(\omega / T)
$$

with

$$
\begin{aligned}
\operatorname{Im}\left[F_{6}(y)\right] & =\int_{0}^{\infty} \frac{d \Omega}{\pi} \operatorname{Im}\left[F_{5}(\Omega)\right] \\
& \times(2 n(\Omega)+f(\Omega-y)+f(\Omega+y))
\end{aligned}
$$

a relationship similar to (6.13). Note again the local nature of the self energy. As the expression (A8) scatters the electron by large momenta around the Fermi surface, there is little difference between single-particle and transport scattering rates, and the latter can be deduced directly from (A9). It should also be noted here that the present perturbative computation for $\Sigma$ is known to break down in certain cases: this was pointed out by Parcollet and Georges 61 who showed that the electron self energy was far more singular than (A9) for the spin glass models of Ref. 61, 64, 67 which had $\mu=0$. Nevertheless, the local nature of the self energy, and $\omega / T$ scaling was preserved.

\section{APPENDIX B: COMPUTATIONS FOR RG EQUATIONS}

This appendix will present some further details of the derivation of the renormalization group equations for Case B, described by the action $\mathcal{S}_{x y}+\mathcal{S}_{I}$. We noted in Section VA that the topological structure of the diagrams is identical to those considered in Ref 49, and so we will not reproduce the diagrams here.

The exponents $\eta$ and $z$ arise from the momentum and frequency dependence of the self energy $\Sigma_{\phi}$ of the $\phi_{1.2}$ field. To leading order, the only such contribution to $\Sigma$ is

$$
\begin{aligned}
\Sigma_{\phi}(q, \omega)=g^{2} \int & \frac{d^{d} p}{(2 \pi)^{d}} \frac{d \Omega}{2 \pi} \frac{|\mathbf{p}|}{\left(|\mathbf{p}|+a_{I}|\Omega|\right)} \\
& \times \frac{1}{\left((\mathbf{p}+\mathbf{q})^{2}+(\omega+\Omega)^{2}+s\right)}
\end{aligned}
$$


where $q=|\mathbf{q}|$. By an expansion of $\Sigma_{\phi}$ for small $q$ and $\omega$, we find

$$
\begin{aligned}
\eta & =\left.\left(-\frac{d}{d q^{2}}\right) \Sigma_{\phi \Lambda}(q, 0)\right|_{q=0} \\
\eta+2(z-1) & =\left.\left(-\frac{d}{d \omega^{2}}\right) \Sigma_{\phi \Lambda}(0, \omega)\right|_{\omega=0} .
\end{aligned}
$$

Here the subscript $\Lambda$ is meant to indicate that the integrals in (B1) are to be carried out as part of a renormalization group procedure. The precise nature of the integral then depends upon the scheme being used:

(i) in the dimensional regularization/minimal subtraction scheme, we perform the integrals in arbitrary dimension $d$, and pick out online the poles in $\epsilon=3-d$ in (B2);

(ii) in the fixed dimension approach 71 , we perform the integral in $d=2$ for a finite $s$, take the derivative of the result with respect to $\sqrt{s}$, and set $s=1$.

(iii) in the soft-cutoff approach 72 , w3, we perform the integrals with a smooth cutoff on the scale $\Lambda$, take the $\Lambda$ derivative, and then set $\Lambda=1$.

Each of these approaches yields closely related expressions for $\mathcal{J}\left(a_{I}\right)$ and $\mathcal{K}\left(a_{I}\right)$.

The remaining terms in (5.3) arise from simple oneloop integrals which can be evaluated at zero external momenta and frequency; these yield the expression

$$
\mathcal{I}\left(m, a_{I}\right)=\int_{\Lambda} \frac{d^{d} p}{(2 \pi)^{d}} \frac{d \Omega}{2 \pi} \frac{|\mathbf{p}|^{m}}{\left(|\mathbf{p}|+a_{I}|\Omega|\right)^{m}\left(p^{2}+\Omega^{2}+s\right)^{2}}
$$

Again the $\Lambda$ subscript indicates that the integrals are to be evaluated in one of the renormalization group schemes discussed below (B2).

We can now present our explicit results for $\mathcal{I}\left(n, a_{I}\right)$, $\mathcal{J}\left(a_{I}\right)$, and $\mathcal{K}\left(a_{I}\right)$, for the respective schemes.

In the dimensional regularization approach, picking poles in $\epsilon=3-d$, we obtained

$$
\begin{aligned}
\mathcal{I}(0, a) & =\frac{1}{8 \pi^{2}} \\
\mathcal{I}(1, a) & =\frac{4 a^{3} \ln a-2 a^{3}+3 \pi a^{2}-2 a+\pi}{8 \pi^{3}\left(1+a^{2}\right)^{2}} \\
\mathcal{I}(2, a) & =\frac{4 a^{5}-3 \pi a^{4}+16 a^{3} \ln a+6 \pi a^{2}-4 a+\pi}{8 \pi^{3}\left(1+a^{2}\right)^{3}} \\
\mathcal{J}(a) & =\frac{(3-2 \ln a) a^{5}-3 \pi a^{4}+(2+6 \ln a) a^{3}+\pi a^{2}-a}{12 \pi^{3}\left(1+a^{2}\right)^{3}} \\
\mathcal{K}(a) & =-3 \mathcal{J}(a)
\end{aligned}
$$

where we have dropped the subscript $I$ on $a$ for brevity.

Similar expressions were obtained in the fixeddimension approach in $d=2$ :

$$
\begin{aligned}
\mathcal{I}(0, a) & =\frac{1}{8 \pi} \\
\mathcal{I}(1, a) & =\frac{(1-a) \sqrt{1+a^{2}}+a^{2} \mathcal{L}(a)}{8 \pi\left(1+a^{2}\right)^{3 / 2}} \\
\mathcal{I}(2, a) & =\frac{\left(a^{3}-2 a^{2}-2 a+1\right) \sqrt{1+a^{2}}+3 a^{2} \mathcal{L}(a)}{8 \pi\left(1+a^{2}\right)^{5 / 2}} \\
\mathcal{J}(a) & =\frac{a\left(2 a^{2}-3 a-1\right) \sqrt{1+a^{2}}-a^{2}\left(a^{2}-2\right) \mathcal{L}(a)}{16 \pi\left(1+a^{2}\right)^{5 / 2}} \\
\mathcal{K}(a) & =-2 \mathcal{J}(a)
\end{aligned}
$$

where

$$
\mathcal{L}(a) \equiv \ln \left(\frac{1+a+\sqrt{1+a^{2}}}{1+a-\sqrt{1+a^{2}}}\right)
$$

We also obtained results in scheme (iii) above, with both soft and hard cutoffs; however we refrain from giving complete expressions as the results are similar to those above.
* Electronic address: subir.sachdev@yale.edu; URL: http: / pantheon.yale.edu/ subir

$\dagger$ Electronic address: takao.morinari@yale.edu; Permanent address: Yukawa Institute for Theoretical Physics, Kyoto University Kyoto 606-8502, Japan
1 M. T. Béal-Monod and K. Maki, Phys. Rev. Lett. 34, 1461 (1975).

2 J. A. Hertz, Phys. Rev. B 14, 1165 (1976).

3 J. Mathon, Proc. R. Soc. London A, 306, 355 (1968).

${ }^{4}$ T. V. Ramakrishnan, Phys. Rev. B 10, 4014 (1974); 
S. G. Mishra and T. V. Ramakrishnan, Phys. Rev. B 18, 2308 (1978); S. G. Mishra and P. A. Sreeram, Phys. Rev. B 57, 2188 (1998).

5 T. Moriya, Spin Fluctuations in Itinerant Electron Magnetism, Springer-Verlag, Berlin (1985); T. Moriya and T. Takimoto, J. Phys. Soc. Jpn. 64, 960 (1995).

${ }^{6}$ G. G. Lonzarich and L. Taillefer, J. Phys. C 18, 4339 (1985); G. G. Lonzarich, in Electron, M. Springford ed., Cambridge University Press, Cambridge (1997).

7 A. J. Millis, Phys. Rev. B, 48, 7183 (1993).

${ }^{8}$ U. Zülicke and A. J. Millis, Phys. Rev. B 51, 8996 (1995).

9 S. Sachdev, A. V. Chubukov, and A. Sokol, Phys. Rev. B 51, 14874 (1995).

10 V. Oganesyan, S. A. Kivelson, and E. Fradkin, Phys. Rev. B 64, 195109 (2001).

11 D. Belitz, T. R. Kirkpatrick, and T. Vojta, Phys. Rev. B 65, 165112 (2002).

12 We should note here a possible exception to this campnical weak coupling scenario: Chubukov and co-workers 13 have studied spin and charge density wave (SDW and CDW) transitions in two dimensions, and have pointed out strong coupling effects in the electron self energy and possibly other observables.

13 Ar. Abanov and A. V. Chubukov Phys. Rev. Lett. 84, 5608 (2000); Ar. Abanov, A. V. Chubukov, and J. Schmalian, cond-mat/0107421.

14 P. Monthoux, A. V. Balatsky, and D. Pines, Phys. Rev. B 46, 14803 (1992); J. Schmalian, D. Pines, and B. Stojkovic, Phys. Rev. Lett. 80, 3839 (1998).

15 C. Castellani, C. Di Castro, and M. Grilli, Phys. Rev. Lett. 75, 4650 (1995); S. Caprara, M. Sulpizi, A. Bianconi, C. Di Castro, and M. Grilli, Phys. Rev. B 59, 14980 (1999); S. Andergassen, S. Caprara, C. Di Castro, and M. Grilli, Phys. Rev. Lett. 87, 056401 (2001); S. Caprara, C. Di Castro, P. Werner, and W. Zwerger, Phys. Rev. Lett. 88, 066403 (2002).

16 S. Sachdev, Quantum Phase Transitions, Cambridge University Press, Cambridge (1999).

17 S. Sachdev, Science 288, 475 (2000).

18 L. Balents, M. P. A. Fisher, and C. Nayak, Int. J. Mod. Phys. B 12, 1033 (1998).

19 M. Vojta, Y. Zhang, and S. Sachdev, Phys. Rev. Lett. 85, 4940 (2000).

20 S. Sachdev and J. Ye, Phys. Rev. Lett. 69, 2411 (1992); A. V. Chubukov and S. Sachdev, Phys. Rev. Lett. 71, 169 (1993); S. Sachdev and M. Vojta, Physica B 280, 333 (2000).

21 O. Parcollet and A. Georges, Phys. Rev. B 59, 5341 (1999).

22 E. Demler, S. Sachdev, and Y. Zhang, Phys. Rev. Lett. 87, 067202 (2001).

23 Y. Zhang, E. Demler, and S. Sachdev, Phys. Rev. B 66, 094501 (2002). This paper discussed mainly the physics of superconductors, and refered to the transition as "exciton fractionalization". In a superconductor, the spin and charge collective modes appear as sharp excitations (because there is no Fermi surface to damp them), and so it is appropriate to refer to the collective modes as quanta of "excitons". In the present paper, we are always dealing with a situation where the spin and charge collective modes are overdamped, and this terminology did not seem appropriate. Otherwise, there is no change in the physical content of the fractionalizaion.

24 J. Zaanen, O. Y. Osman, H. V. Kruis, Z. Nussinov, and J. Tworzydlo, Phil. Mag. B 81, 1485 (2002).
25 J. L. Tallon and J. W. Loram, Physica C 349, 53 (2001).

26 T. Valla, A. V. Fedorov, P. D. Johnson, B. O. Wells, S. L. Hulbert, Q. Li, G. D. Gu, and N. Koshizuka, Science 285, 2110 (1999).

27 P. Coleman, C. Pépin, Q. Si, and R. Ramazashvili, J. Phys: Condens. Matt. 13, 723 (2001) and references therein.

28 C. Pfleiderer, G. J. McMullan, S. R. Julian, and G. G. Lonzarich Phys. Rev. B 55, 8330 (1997); S. A. Grigera, R. S. Perry, A. J. Schofield, M. Chiao, S. R. Julian, G. G. Lonzarich, S. I. Ikeda, Y. Maeno, A. J. Millis, and A. P. Mackenzie, Science 294, 329 (2001); H. v. Löhneysen, C. Pfleiderer, T. Pietrus, O. Stockert, and B. Will, Phys. Rev. B 63, 134411 (2001).

29 A. Schröder, G. Aeppli, R. Coldea, M. Adams, O. Stockert, H.v. Löhneysen, E. Bucher, R. Ramazashvili, and P. Coleman, Nature 407, 351 (2000).

30 T. Senthil and M. P. A. Fisher, Phys. Rev. B 62, 7850 (2000).

31 N. Read and S. Sachdev, Phys. Rev. Lett. 66, 1773 (1991); X.-G. Wen, Phys. Rev. B 44, 2664 (1991).

32 P. E. Lammert, D. S. Rokhsar, and J. Toner, Phys. Rev. Lett. 70, 1650 (1993); Phys. Rev. E 52, 1778 (1995).

33 Section 13.3 .2 in Ref. 16.

34 E. Demler and F. Zhou, Phys. Rev. Lett. 88, 163001 (2002).

35 S. Wakimoto, G. Shirane, Y. Endoh, K. Hirota, S. Ueki, K. Yamada, R. J. Birgeneau, M. A. Kastner, Y. S. Lee, P. M. Gehring, and S. H. Lee, Phys. Rev. B 60, R769 (1999); S. Wakimoto, R. J. Birgeneau, Y. S. Lee, and G. Shirane, Phys. Rev. B 63, 172501 (2001).

36 O. Zachar, S. A. Kivelson, and V. J. Emery, Phys. Rev. B 57, 1422 (1998).

37 A. Polkovnikov, S. Sachdev, and M. Vojta, Proceedings of the 23rd International Conference on Low Temperature Physics, Hiroshima, cond-mat/0208334.

38 E. Brézin and J. Zinn-Justin, Phys. Rev. B 14, 3110 (1976).

39 J. M. Tranquada, J. D. Axe, N. Ichikawa, Y. Nakamura, S. Uchida, and B. Nachumi, Phys. Rev. B 54, 7489 (1996).

40 Y. S. Lee, R. J. Birgeneau, M. A. Kastner, Y. Endoh, S. Wakimoto, K. Yamada, R. W. Erwin, S.-H. Lee, and G. Shirane, Phys. Rev. B 60, 3643 (1999).

41 For commensurate wavevectors, the $S_{1}$ factor is reduced to the discrete space $Z_{p}$, but all of our results continue to apply as long as $p>2$.

42 F. Wegner, J. Math. Phys. 12, 2259 (1971).

43 E. Fradkin and S. H. Shenker, Phys. Rev. D 19, 3682 (1979).

44 A. Aharony in Phase Transitions and Critical Phenomena, vol 6, C. Domb and M. S. Green eds., Academic Press, London (1976).

45 P. Calabrese, A. Pelissetto, and E. Vicari, Phys. Rev. E 65, 046115 (2002).

46 M. Campostrini, M. Hasenbusch, A. Pelissetto, P. Rossi, and E. Vicari, Phys. Rev. B 63, 214503 (2001).

47 M. A. de Moura, T. C. Lubensky, Y. Imry, and A. Aharony, Phys. Rev. B 13, 2176 (1976).

48 M. Campostrini, A. Pelissetto, P. Rossi, and E. Vicari, Phys. Rev. E 60, 3526 (1999); 65, 066127 (2002).

49 E. Frey and L. Balents, Phys. Rev. B 55, 1050 (1997).

50 A. Aharony, Phys. Rev. B 8, 4270 (1973).

51 B. I. Halperin, P. C. Hohenberg, and S.-k. Ma, Phys. Rev. B 10, 139 (1974).

52 C. M. Varma, Z. Nussinov, and W. van Saarloos, Phys. 
Rep. 361, 267 (2002).

53 Q. Si, S. Rabello, K. Ingersent, and J. L. Smith, Nature 413, 804 (2001) and cond-mat/0202414.

${ }^{54}$ L. Zhu and Q. Si, Phys. Rev. B 66, 024426 (2002).

55 S. Burdin, D. R. Grempel, and A. Georges, Phys. Rev. B 66, 045111 (2002).

56 G. Zarand and E. Demler, Phys. Rev. B 66, 024427 (2002).

57 S Burdin, M. Grilli, and D. R. Grempel, cond$\mathrm{m}$ at/0206174.

58 A renormalization group argument on the implications of a divergent local susceptibility in impurity models runs as follows. Consider an impurity model with a local spin $S_{1 \alpha}$ with a local susceptibility $\chi_{\text {loc }}=\int d \tau\left\langle S_{1 \alpha}(\tau) S_{1 \alpha}(0)\right\rangle \sim$ $T^{-\gamma}$ with $\gamma>0$. Then power counting implies $\operatorname{dim}\left[S_{1 \alpha}\right]=$ $(1-\gamma) z / 2$. Now bring in a second identical impurity model, with spin $S_{2 \alpha}$ initially decoupled from $S_{1 \alpha}$, which also has $\operatorname{dim}\left[S_{2 \alpha}\right]=(1-\gamma) z / 2$. Finally, turn on an infinitesimal coupling $K \int d \tau S_{1 \alpha}(\tau) S_{2 \alpha}(\tau)$ between the two impurities; scaling implies $\operatorname{dim}[K]=z \gamma>0$, and so $K$ is a relevant perturbation. A possible escape from this instability is that $K$ is already accounted for in the 'baths' of the two impurity models, but this has to be shown beyond mean-field theory. An analogy with the sliding Luttinger liquid (C. S. O'Hern, T. C. Lubensky, and J. Toner, Phys. Rev. Lett. 83, 2745 (1999), A. Vishwanath and D. Carpentier, Phys. Rev. Lett. 86, 676 (2001), and V. J. Emery, E. Fradkin, S. A. Kivelson, and T. C. Lubensky Phys. Rev. Lett. 85, 2160 (2000)), another situation with apparently lower dimensional criticality embedded in a higher dimensional space, might be helpful: in this case there is only a marginal coupling between the Luttinger liquids, but this does change all the exponents in the full theory.

59 B. L. Altshuler, L. B. Ioffe, and A. J. Millis, Phys. Rev. B 50, 14048 (1994).

60 C. Castellani, S. Caprara, C. Di Castro and A. Maccarone, Nucl. Phys. B 594, 747 (2001).

61 O. Parcollet and A. Georges, Phys. Rev. B 59, 5341 (1999).

${ }^{62}$ C. Howald, H. Eisaki, N. Kaneko, and A. Kapitulnik, condmat/0201546.

${ }^{63}$ C. J. Halboth and W. Metzner, Phys. Rev. B 61, 7364 (2000); R. Shankar, Rev. Mod. Phys. 66, 129 (1994).

64 S. Sachdev and J. Ye, Phys. Rev. Lett. 70, 3339 (1993).

65 S. Sachdev, N. Read, and R. Oppermann, Phys. Rev. B, 52, 10286 (1995).

66 A. M. Sengupta and A. Georges, Phys. Rev. B 52, 10295 (1995).

67 A. Georges, O. Parcollet, and S. Sachdev, Phys. Rev. Lett. 85, 840 (2000); Phys. Rev. B 63, 134406 (2001).

68 G. Biroli and O. Parcollet Phys. Rev. B 65, 094414 (2002).

69 L. Arrachea and M. J. Rozenberg, Phys. Rev. Lett. 86, 5172 (2001); Phys. Rev. B 65, 224430 (2002).

70 D. Dalidovich and V. Dobrosavljevic, Phys. Rev. B 66, 081107 (2002).

71 G. Parisi, Statistical Field Theory, Perseus Books, Reading, Massachusetts (1988).

72 E. Brézin, J. C. Le Guillou, J. Zinn-Justin in Phase Transitions and Critical Phenomena, vol 6, C. Domb and M. S. Green eds, Academic Press, London (1976); see Section V.

73 M. Vojta, Y. Zhang, and S. Sachdev, Int. J. Mod. Phys. B 14, 3719 (2000). 